Supporting Information

\title{
Generation and Synthetic Application of Trifluoromethyl Diazomethane Utilizing Continuous Flow Technologies
}

Bartholomäus Pieber* and C. Oliver Kappe*

Institute of Chemistry, University of Graz, NAWI Graz, Heinrichstrasse 28, A-8010 Graz, Austria

bartholomaues.pieber@uni-graz.at,oliver.kappe@uni-graz.at

\section{Table of Contents}

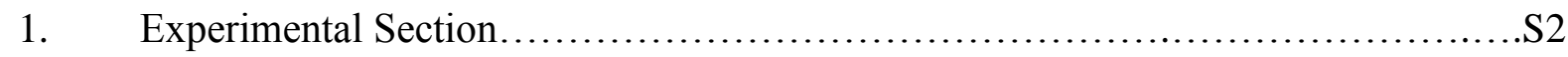

2. Continuous preparation of anhydrous Trifluoromethyl Diazomethane (2)..........S2

3. Continuous preparation and utilization of $\mathbf{2}$ in a multistep approach............S4-S11

4. X-ray Crystallography.................................................. 11

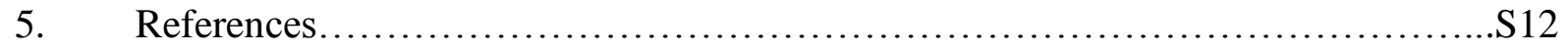

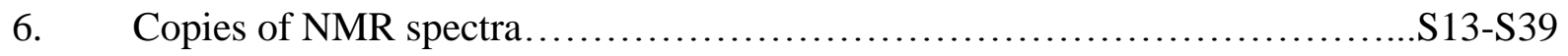




\section{Experimental Section}

General Remarks. All compounds were obtained from standard commercial vendors and used without further purification. NMR spectra were recorded on a $300 \mathrm{MHz}$ instrument using DMSO- $\mathrm{d}_{6}$ as solvent. Chemical shifts $(\delta)$ are expressed in ppm downfield from TMS as internal standard. The letters $\mathrm{s}, \mathrm{d}, \mathrm{t}, \mathrm{q}$ and $\mathrm{m}$ are used to indicate a singlet, doublet, triplet, quadruplet, and multiplet, respectively. GC-FID analysis was performed on a Trace-GC (ThermoFisher) with a flame ionization detector using a HP5 column (30 $\mathrm{m} \times 0.250 \mathrm{~mm} \times 0.025$ $\mu \mathrm{m})$. After $1 \mathrm{~min}$ at $50^{\circ} \mathrm{C}$ the temperature was increased in $25^{\circ} \mathrm{C} \mathrm{min}{ }^{-1}$ steps up to $300^{\circ} \mathrm{C}$ and kept at $300^{\circ} \mathrm{C}$ for $4 \mathrm{~min}$. The detector gas for the flame ionization is $\mathrm{H}_{2}$ and compressed air (5.0 quality). Melting points were determined on a standard melting point apparatus. Silica gel flash chromatography separations were performed on a Biotage SP1 instrument using toluene/ethyl acetate mixtures (containing $1 \%(\mathrm{v} / \mathrm{v})$ triethylamine) as eluent. HRMS experiments were performed on a GCT Premier spectrometer (Waters, EI, 70eV, ion source at $200{ }^{\circ} \mathrm{C}$ ) by direct injection. X-ray diffraction measurements were performed on a standard CCD diffractometer by using graphite monochromatized Mo K $\alpha$ radiation.

\section{Continuous Preparation of Anhydrous Trifluoromethyl Diazomethane (2).}

General Procedure. For feed A 2,2,2-trifluoroethylamine hydrochloride (1, 1.69 g, 12.5 mmol) was dissolved in water $(25 \mathrm{~mL}, 0.5 \mathrm{M})$ and two drops of concentrated $\mathrm{H}_{2} \mathrm{SO}_{4}$ were added. For feed $\mathrm{B}, \mathrm{NaNO}_{2}(1.73 \mathrm{~g}, 25 \mathrm{mmol})$ was dissolved in water $(25 \mathrm{~mL}, 1 \mathrm{M})$. Feed A and $\mathrm{B}$ were pumped into a T-mixer at room temperature by two syringe pumps (Asia,

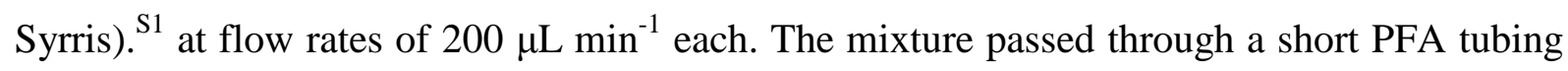
then further through the inner tube of the commercially available tube-in-tube device (GAM II, Uniqsis) ${ }^{\mathrm{S} 2}$ made out of gas permeable Teflon AF2400 (0.8 mm inner diameter, $2 \mathrm{~mL}$ ). The aqueous waste stream leaving the inner tube of the reactor was quenched into $2 \mathrm{M} \mathrm{H}_{2} \mathrm{SO}_{4}$ after passing a 7 bar back pressure regulator (Vapourtec). ${ }^{\mathrm{S} 3}$ The outer tube of the reactor made out of a gas impermeable perfluoroalkox polymer (1.6 mm inner diameter, $5 \mathrm{~mL})$ was connected to a third syringe pump carrying feed C (dry THF) at a flow rate of $100 \mu \mathrm{L} / \mathrm{min}$. The product stream from the outer tube was collected in a cooled flask after depressurization in a 6 bar backpressure regulator. To estimate the amount of generated and separated trifluoromethyl diazomethane, the collected mixture was transferred into an inverted measuring cylinder filled with aqueous $\mathrm{H}_{2} \mathrm{SO}_{4}\left(\begin{array}{ll}1 & \text { M) }\end{array}\right.$ (see Figure $\mathrm{S} 2$ ). There decomposition of 2 generates stoichiometric amounts of nitrogen gas $\left(102 \mathrm{~mL} \hat{=} 4.1 \mathrm{mmol}\right.$ at $17{ }^{\circ} \mathrm{C}$ and 761.3 torr $)$ indicating a yield of $\sim 33 \%$ of the desired diazo compound 2 . 

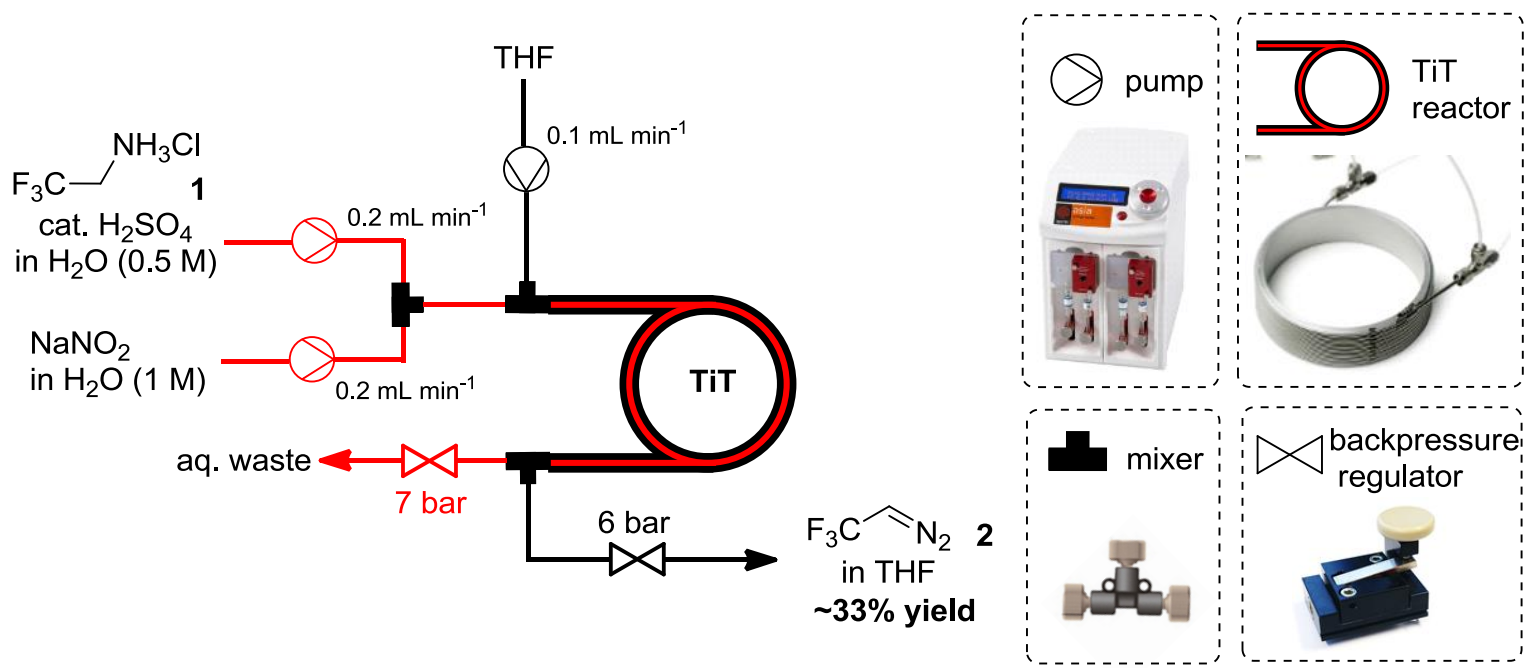

Figure S1. Detailed description of the continuous flow reactor and conditions for the synthesis of trifluoromethyl diazomethane (2).

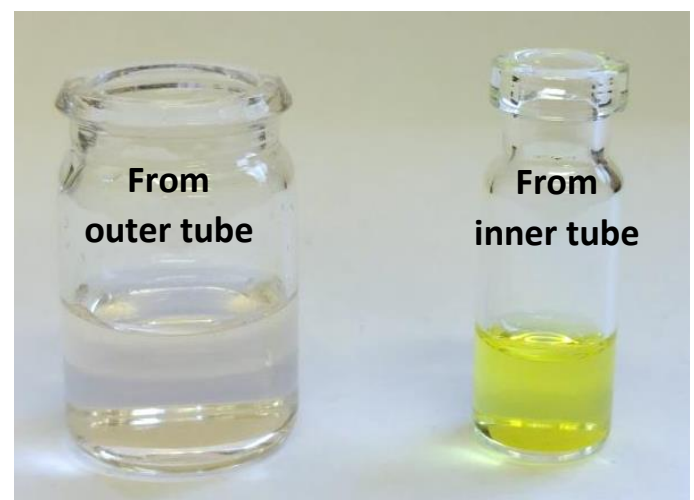

Figure S3. Picture of samples collected from the continuous synthesis and purification of trifluoromethyl diazomethane (2). The aqueous mixture leaving the outer tube (left) is almost colorless whereas the organic solution leaving the inner tube (right) is strongly yellow colored.

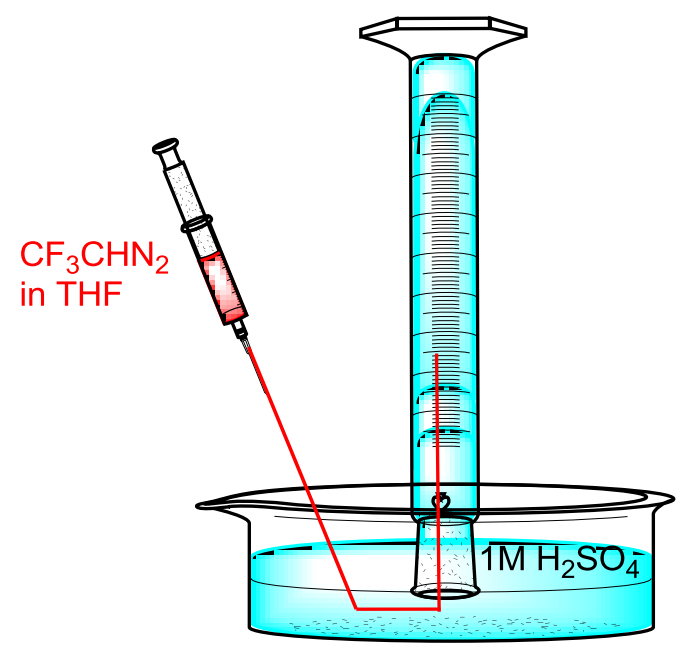

Figure S2. Experimental setup for the quantification of trifluoromethyl diazomethane (2) by decomposition in an acidic solution. 


\section{Continuous Preparation and Utilization of 2 in a Multistep Approach}

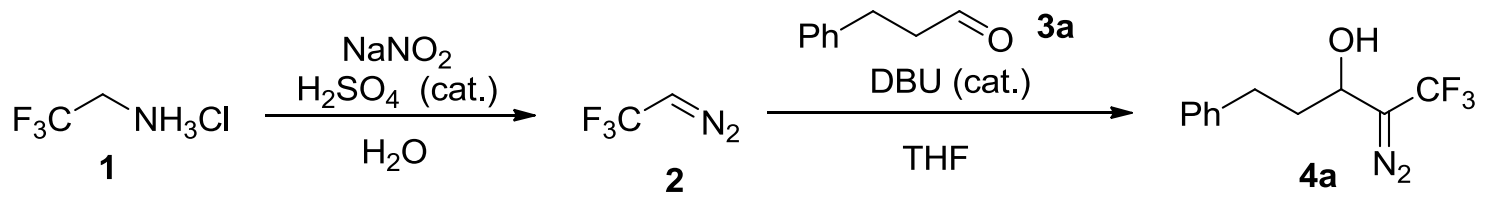

Scheme S1. Model reaction for the two step process: synthesis of trifluoromethyl diazomethane (2) from 2,2,2-trifluoroethylamine hydrochloride (1) and its subsequent utilization in a DBU catalyzed aldol reaction with 3-phenylpropanal.

Analysis of the Model Reaction by GC-FID. A typical GC-FID chromatogram from the DBU catalyzed reaction of 3-phenylpropanal (3a) with trifluoromethyl diazomethane (2) contains 5 major peaks (Figure S4-A). The only peak that can be clearly assigned (5.97 min) corresponds to unreacted starting material (3a). Figure S4-B shows a GC-FID chromatogram of isolated 2-diazo-1,1,1-trifluoro-5-phenylpentan-3-ol (4a). The title compound apparently decomposes in the injector block resulting in four degradation products $(5.67,5.97,6.15,6.32$ min). Thus, peak area integration of the degradation products and the starting material (3a) was used to determine the conversion for optimization studies.

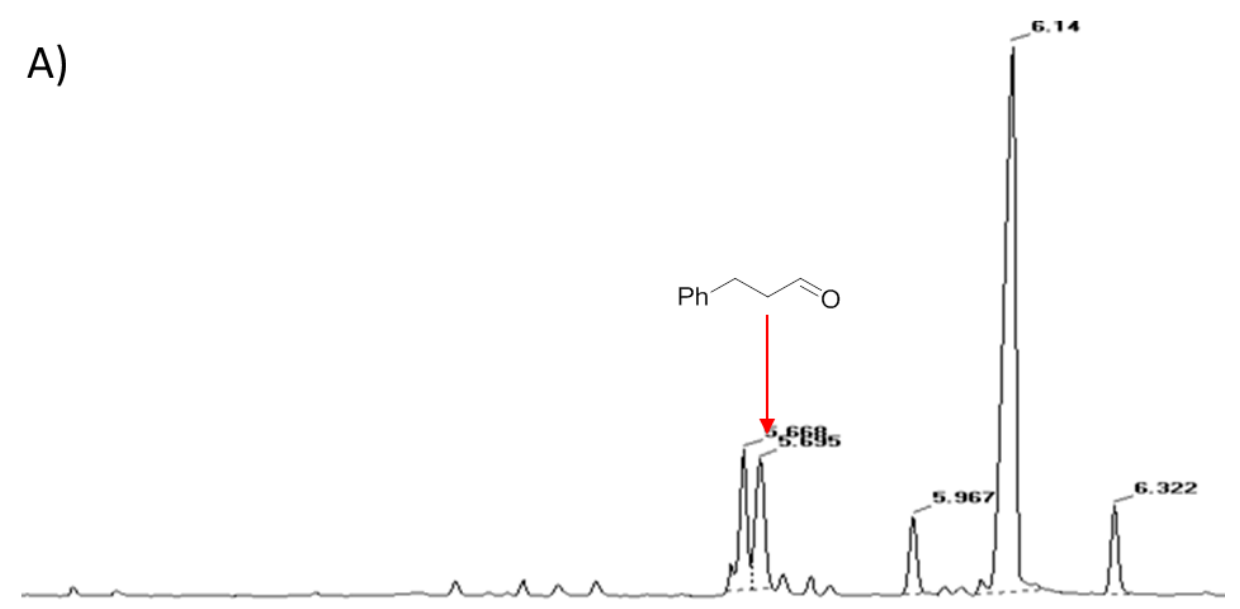

B)

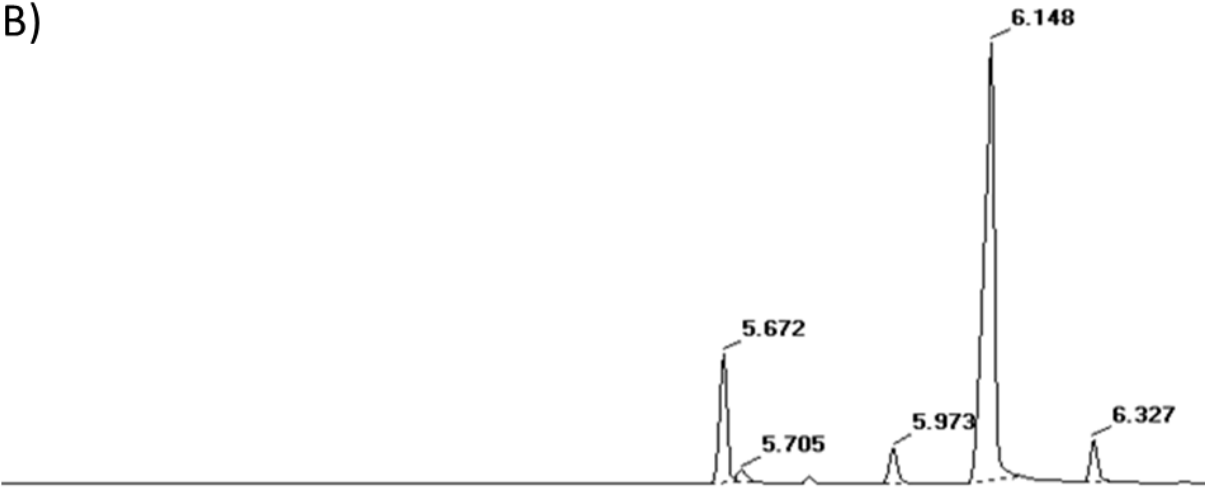

Figure S4. GC-FID chromatogram of the crude reaction mixture (A) and of the isolated diazo pure compound $4 \mathbf{a}(\mathrm{B})$ 
Table S1. Optimization study for the two-step process by homogeneous catalysis. ${ }^{a}$

\begin{tabular}{|c|c|c|c|c|c|c|}
\hline & $\begin{array}{l}\mathrm{F}_{3} \mathrm{NH}_{3} \mathrm{Cl} \\
\text { cat. } \mathrm{H}_{2} \mathrm{SO}_{4} \\
\text { in } \mathrm{H}_{2} \mathrm{O}(0.5 \mathrm{M}) \\
\mathrm{NaNO}_{2} \\
\text { in } \mathrm{H}_{2} \mathrm{O}(1 \mathrm{M}) \\
\text { aq. was }\end{array}$ & $\mathrm{dA}$ & feed & гіт & & $\prod_{\mathrm{N}_{2}}^{\mathrm{H}} \mathrm{CF}_{3}$ \\
\hline entry & $\begin{array}{c}\text { flow rate feed } \\
\mathrm{A} / \mathrm{B} / \mathrm{C}\left[\mathrm{mL} \mathrm{min}^{-1}\right]\end{array}$ & 3a $[\mathbf{M}]$ & $\begin{array}{c}\text { DBU } \\
{[\mathrm{mol} \%]}\end{array}$ & $\begin{array}{c}\mathbf{t} \\
{[\mathrm{min}]}\end{array}$ & $\begin{array}{c}\mathbf{T} \\
{\left[{ }^{\circ} \mathbf{C}\right]}\end{array}$ & conversion $[\%]^{b}$ \\
\hline 1 & $0.2 / 0.2 / 0.1$ & 0.8 & 10 & 60 & r.t. & 50 \\
\hline 2 & $0.2 / 0.2 / 0.2$ & 0.2 & 10 & 30 & r.t. & 37 \\
\hline 3 & $0.2 / 0.2 / 0.2$ & 0.4 & 10 & 30 & r.t. & 43 \\
\hline $4^{\mathrm{c}}$ & $0.2 / 0.2 / 0.2$ & 0.4 & 10 & 30 & r.t. & 46 \\
\hline $5^{\mathrm{d}}$ & $0.2 / 0.2 / 0.2$ & 0.4 & 10 & 30 & r.t. & 18 \\
\hline $6^{\mathrm{e}}$ & $0.2 / 0.2 / 0.2$ & 0.4 & 10 & 30 & r.t. & 12 \\
\hline 8 & $0.2 / 0.2 / 0.2$ & 0,8 & 10 & 30 & r.t. & 33 \\
\hline 9 & $0.2 / 0.2 / 0.2$ & 0.4 & 10 & 30 & 50 & 54 \\
\hline 10 & $0.2 / 0.2 / 0.2$ & 0.4 & 10 & 30 & 80 & 41 \\
\hline 11 & $0.2 / 0.2 / 0.2$ & 0.4 & 5 & 30 & 50 & 28 \\
\hline 12 & $0.2 / 0.2 / 0.2$ & 0.4 & 20 & 30 & 50 & 35 \\
\hline 13 & $0.2 / 0.2 / 0.1$ & 0.4 & 10 & 60 & 50 & 58 \\
\hline 14 & $0.2 / 0.2 / 0.1$ & 0.4 & 20 & 60 & 50 & 65 \\
\hline 15 & $0.2 / 0.2 / 0.1$ & 0.4 & 40 & 60 & 50 & 39 \\
\hline 16 & $0.2 / 0.2 / 0.1$ & 0.3 & 20 & 60 & 50 & 66 \\
\hline 17 & $0.1 / 0.1 / 0.1$ & 0.4 & 20 & 60 & 50 & 52 \\
\hline 18 & $0.2 / 0.2 / 0.1$ & 0.4 & 20 & 60 & 50 & 65 \\
\hline 19 & $0.3 / 0.3 / 0.1$ & 0.4 & 20 & 60 & 50 & 67 \\
\hline 20 & $0.2 / 0.2 / 0.075$ & 0.4 & 20 & 80 & 50 & 73 \\
\hline 21 & $0.2 / 0.2 / 0.075$ & 0.4 & 20 & 120 & 50 & 80 \\
\hline
\end{tabular}

${ }^{a}$ Reactions were carried out on a $2.4 \mathrm{mmol}$ scale. ${ }^{\mathrm{b}}$ Conversion was determined as GC-FID peak area percent under steady state conditions. ${ }^{\mathrm{c}} \mathrm{MeCN}$ was used as solvent in the outer tube. ${ }^{\mathrm{d}}$ Toluene was used as solvent in the outer tube. ${ }^{\mathrm{e}} \mathrm{MeOH}$ was used as solvent in the outer tube 
Table S2. Optimization study for the two-step process by heterogeneous catalysis. ${ }^{\text {a }}$

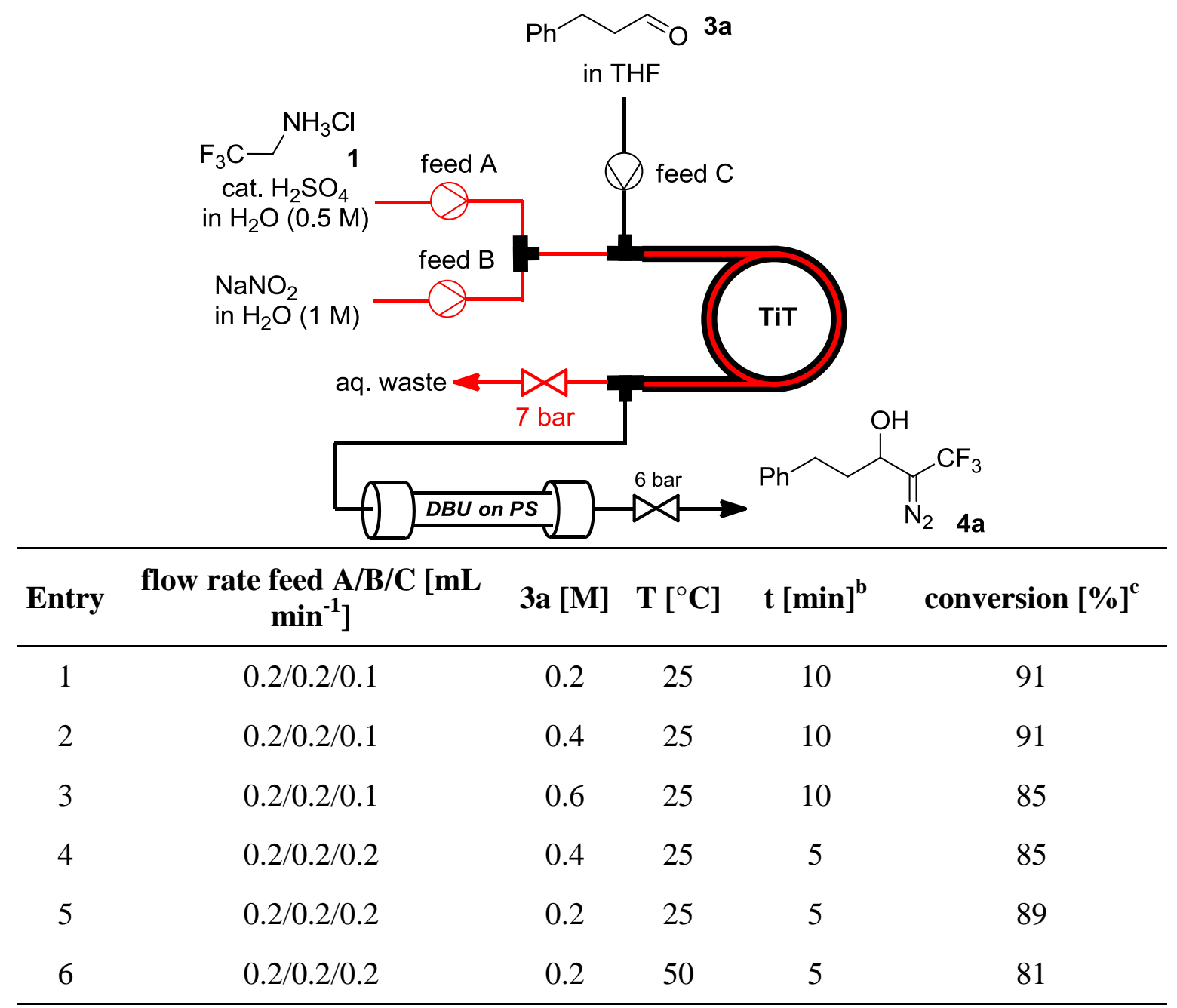

${ }^{a}$ Reactions were carried out on a 2.4 mmol scale. For the packed-bed reactor, an Omnifit glass column (6.6 $\mathrm{mm}$ i.d., $2.4 \mathrm{~mL}$ volume) was filled with 1.2-1.3 g polymer-bound DBU. ${ }^{\mathrm{b}}$ Time of the reaction mixture inside the packed-bed reactor ${ }^{\text {c }}$ Conversion was determined as GC-FID peak area percent under steady state conditions.

Catalyst Reactivation. The immobilized catalyst was regenerated after 2-3 reactions in order to guarantee reproducible results. Thus, a solution of DBU in THF $(0.5 \mathrm{M})$ was pumped through the packed-bed reactor at $0.2 \mathrm{~mL} \mathrm{~min}^{-1}$. Before reinstalling the cartridge, pure THF $(10 \mathrm{~mL})$ was pumped through the reactor $\left(0.2 \mathrm{~mL} \mathrm{~min}^{-1}\right)$ to remove all traces of the homogeneous base. 


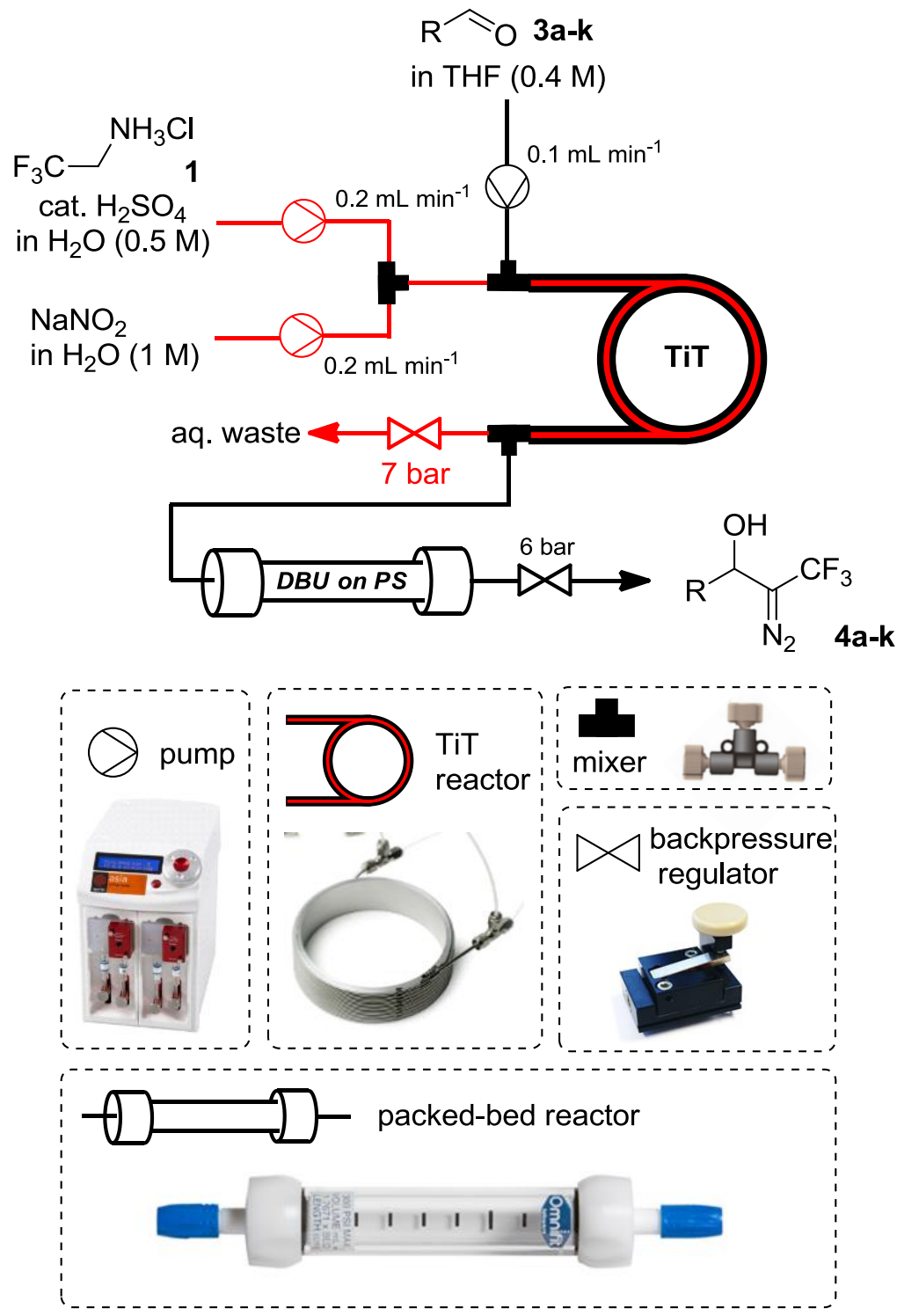

Figure S5. Detailed description of the continuous flow reactor including optimized conditions for the synthesis of trifluoromethyl diazomethane (2) and its subsequent utilization in the heterogeneously catalyzed condensation with aldehydes. 
General Procedure for the Generation of Trifluoromethyl Diazomethane (2) and its Subsequent Condensation with Aldehydes using Heterogeneous Catalysis under Optimized Conditions (Table 1). For feed A 2,2,2-trifluoroethylamine hydrochloride (1, $3.39 \mathrm{~g}, 25 \mathrm{mmol})$ was dissolved in water $(50 \mathrm{~mL}, 0.5 \mathrm{M})$ and four drops of concentrated $\mathrm{H}_{2} \mathrm{SO}_{4}$ were added. For feed $\mathrm{B}, \mathrm{NaNO}_{2}(3.45 \mathrm{~g}, 50 \mathrm{mmol})$ was dissolved in water $(50 \mathrm{~mL}, 1$ $\mathrm{M})$. Feeds $\mathrm{A}$ and $\mathrm{B}$ were pumped into a T-mixer at room temperature by two syringe pumps (Asia, Syrris). ${ }^{\mathrm{S} 1}$ at flow rates of $200 \mu \mathrm{L} \mathrm{min}{ }^{-1}$ each. The mixture passed through a short PFA tubing then further through the inner tube of the commercially available tube-in-tube device (GAM II, Uniqsis) $^{\text {S2 }}$ made out of gas permeable Teflon AF2400 (0.8 mm inner diameter, $2 \mathrm{~mL}$ ). The aqueous waste stream leaving the inner tube of the reactor was quenched into $2 \mathrm{M}$ $\mathrm{H}_{2} \mathrm{SO}_{4}$ after passing a 7 bar back pressure regulator (Vapourtec ${ }^{\mathrm{S} 3}$. The outer tube of the reactor made out of a gas impermeable perfluoroalkox polymer (1.6 $\mathrm{mm}$ inner diameter, $\sim 6$ $\mathrm{mL}$ ) was connected to a third syringe pump carrying feed C (dry THF) at a flow rate of 100 $\mu \mathrm{L} / \mathrm{min}$. The outlet of the outer tubing was connected to a glass column (Omnifit, $6.6 \mathrm{~mm}$ i.d., $2.4 \mathrm{~mL}$ volume) which was packed with 1.2-1.3 g polymer-bound DBU (Sigma-Aldrich). After around 30 min equilibration time, feed $\mathrm{C}$ was switched form pure solvent to a $0.4 \mathrm{M}$ solution of the respective aldehyde $(\mathbf{3 a}-\mathbf{k}, 2.4 \mathrm{mmol})$ in dry THF $(6 \mathrm{~mL})$. The product stream was collected after depressurization in a 6 bar backpressure regulator. The crude reaction mixture was concentrated in vacuum and purified by flash column chromatography using toluene/ethyl acetate mixtures (containing $1 \%(\mathrm{v} / \mathrm{v})$ triethylamine) as eluent.

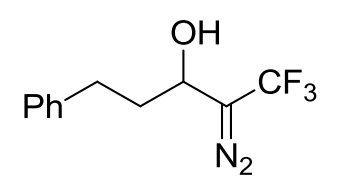

2-Diazo-1,1,1-trifluoro-5-phenylpentan-3-ol (Table 1, 4a). Prepared from 3-phenylpropanal (3a, $317 \mu \mathrm{L}, 2.4 \mathrm{mmol})$ according to the general procedure. Isolation by column chromatography afforded the title compound in $76 \%$ yield $(443.2 \mathrm{mg}, 1.81 \mathrm{mmol})$ as a yellow oil. ${ }^{1} \mathrm{H}$ NMR (300 MHz, DMSO) $\delta 7.25-7.16(\mathrm{~m}, 2 \mathrm{H}), 7.16-7.07(\mathrm{~m}, 3 \mathrm{H}), 5.73(\mathrm{~d}, J=6.2 \mathrm{~Hz}, 1 \mathrm{H}), 4.22(\mathrm{dt}, J=7.6,6.2 \mathrm{~Hz}$, $1 \mathrm{H}), 2.70-2.49(\mathrm{~m}, 2 \mathrm{H}), 1.90-1.67(\mathrm{~m}, 2 \mathrm{H}) .{ }^{13} \mathrm{C}$ NMR (75 MHz, DMSO) $\delta 141.48,128.81$, 128.72, 126.36, 120.00 (q, $J=268.6 \mathrm{~Hz}), 55.53$ (q, $J=41.0 \mathrm{~Hz}), 37.08,31.59 .{ }^{19} \mathrm{~F}$ NMR $(282$ MHz, DMSO) $\delta-55.85$. HRMS (EI): $m / z$ : calcd for $\mathrm{C}_{11} \mathrm{H}_{11} \mathrm{OF}_{3}\left[\mathrm{M}-\mathrm{N}_{2}\right]^{+}: 216.0762$, found: 216.0769 .<smiles>OC(Cc1ccccc1)C(=[W])C(F)(F)F</smiles>

3-Diazo-4,4,4-trifluoro-1-phenylbutan-2-ol (Table 1, 4b). Prepared from 2-phenylacetaldehyde (3b, $267 \mu \mathrm{L}, 2.4 \mathrm{mmol})$ according to the general procedure. Isolation by column chromatography afforded the title 
compound in $46 \%$ yield $(255.0 \mathrm{mg}, 1.11 \mathrm{mmol})$ as a yellow oil. ${ }^{1} \mathrm{H}$ NMR (300 MHz, DMSO$\left.d_{6}\right) \delta 7.32-7.20(\mathrm{~m}, 5 \mathrm{H}), 5.92(\mathrm{~d}, J=5.9 \mathrm{~Hz}, 1 \mathrm{H}), 4.64-4.51(\mathrm{~m}, 1 \mathrm{H}), 3.02-2.80(\mathrm{~m}, 2 \mathrm{H})$. ${ }^{13} \mathrm{C}$ NMR (75 MHz, DMSO) $\delta 137.48,129.62,128.67,126.96,126.90$ (q, $\left.J=268.1 \mathrm{~Hz}\right)$, 66.20, $55.36(\mathrm{q}, J=43.3 \mathrm{~Hz}), 41.53 .{ }^{19} \mathrm{~F}$ NMR (282 MHz, DMSO) $\delta-56.04$. HRMS (EI): $m / z$ : calcd for $\mathrm{C}_{10} \mathrm{H}_{9} \mathrm{OF}_{3}\left[\mathrm{M}-\mathrm{N}_{2}\right]^{+}$: 202.0605, found: 202.0610 .<smiles>CC(C)(C)C(C(O)C#N)C(F)(F)F</smiles>

2-Diazo-1,1,1-trifluorodecan-3-ol (Table 1, 4c). Prepared from octanal (3c, $375 \mu \mathrm{L}, 2.4 \mathrm{mmol}$ ) according to the general procedure. Isolation by column chromatography afforded the title compound in $71 \%$ yield $(405.9 \mathrm{mg}, 1.70$ mmol) as yellow oil. ${ }^{1} \mathrm{H}$ NMR $\left(300 \mathrm{MHz}, \mathrm{DMSO}-d_{6}\right) \delta 5.67(\mathrm{~d}, J=6.1 \mathrm{~Hz}, 1 \mathrm{H}), 4.35-4.21$ $(\mathrm{m}, 1 \mathrm{H}), 1.67-1.45(\mathrm{~m}, 2 \mathrm{H}), 1.25(\mathrm{~s}, 10 \mathrm{H}), 0.89-0.81(\mathrm{~m}, 3 \mathrm{H}) .{ }^{13} \mathrm{C} \mathrm{NMR}(176 \mathrm{MHz}$, DMSO) $\delta 126.57(\mathrm{q}, J=268.2 \mathrm{~Hz}), 64.42,54.85$ (q, $J=40.8 \mathrm{~Hz}), 37.76,31.13,28.55,28.51$, 24.89, 22.01, 13.86. ${ }^{19} \mathrm{~F}$ NMR (282 MHz, DMSO) $\delta-56.11$. HRMS (EI): $m / z$ : calcd for $\mathrm{C}_{10} \mathrm{H}_{17} \mathrm{OF}_{3}\left[\mathrm{M}-\mathrm{N}_{2}\right]^{+}:$210.1232, found: 210.1248 .<smiles>N#CC(C(O)C1CCCCC1)C(F)(F)F</smiles>

1-Cyclohexyl-2-diazo-3,3,3-trifluoropropan-1-ol (Table 1, 4d). Prepared from cyclohexanecarbaldehyde $(\mathbf{3 d}, 291 \mu \mathrm{L}, 2.4 \mathrm{mmol})$ according to the general procedure. Isolation by column chromatography afforded the title compound in $65 \%$ yield (349.2 mg, $1.57 \mathrm{mmol}$ ) as yellow oil. ${ }^{1} \mathrm{H}$ NMR (300 MHz, DMSO$\left.d_{6}\right) \delta 5.73(\mathrm{~d}, J=5.9 \mathrm{~Hz}, 1 \mathrm{H}), 3.94(\mathrm{dd}, J=8.1,6.0 \mathrm{~Hz}, 1 \mathrm{H}), 1.98-1.85(\mathrm{~m}, 1 \mathrm{H}), 1.78-1.66$ $(\mathrm{m}, 2 \mathrm{H}), 1.65-1.54(\mathrm{~m}, 2 \mathrm{H}), 1.49-1.37(\mathrm{~m}, 1 \mathrm{H}), 1.27-0.92(\mathrm{~m}, 5 \mathrm{H}) .{ }^{13} \mathrm{C}$ NMR $(75 \mathrm{MHz}$, DMSO) $\delta 127.10(\mathrm{q}, J=268.0 \mathrm{~Hz}), 121.77,121.74,69.35,54.41(\mathrm{q}, J=41.3 \mathrm{~Hz}), 42.47$, 29.06, 28.91, 26.30, 25.87, 25.71. ${ }^{19} \mathrm{~F}$ NMR (282 MHz, DMSO) $\delta$-55.92. HRMS (EI): $m / z$ : calcd for $\mathrm{C}_{9} \mathrm{H}_{13} \mathrm{OF}_{3}\left[\mathrm{M}-\mathrm{N}_{2}\right]^{+}:$194.0918, found: 194.0922 .<smiles>OC(COc1ccccc1)C(=[W])C(F)(F)F</smiles>

1-(Benzyloxy)-3-diazo-4,4,4-trifluorobutan-2-ol (Table 1, 4e). Prepared from 2-(benzyloxy)acetaldehyde (3e, $343 \mu \mathrm{L}, 2.4 \mathrm{mmol})$ according to the general procedure. Isolation by column chromatography afforded the title compound in $72 \%$ yield (447.9 mg, $1.72 \mathrm{mmol}$ ) as yellow oil. ${ }^{1} \mathrm{H}$ NMR (300 MHz, DMSO$\left.d_{6}\right) \delta 7.40-7.26(\mathrm{~m}, 5 \mathrm{H}), 5.95(\mathrm{~d}, J=5.9 \mathrm{~Hz}, 1 \mathrm{H}), 4.57-4.45(\mathrm{~m}, 3 \mathrm{H}), 3.68-3.43(\mathrm{~m}, 2 \mathrm{H})$. ${ }^{13} \mathrm{C}$ NMR (75 MHz, DMSO) $\delta 138.42,128.73,128.02,127.98,127.10$ (q, $J=268.2 \mathrm{~Hz}$ ), 72.82, 71.73, 64.13, 54.32 (q, $J=41.5 \mathrm{~Hz}) .{ }^{19} \mathrm{~F}$ NMR (282 MHz, DMSO) $\delta-55.96$. HRMS (EI): $m / z$ : calcd for $\mathrm{C}_{11} \mathrm{H}_{11} \mathrm{O}_{2} \mathrm{~F}_{3}\left[\mathrm{M}-\mathrm{N}_{2}\right]^{+}: 232.0711$, found: 232.0712 . 
$\prod_{\mathrm{N}_{2}}^{\mathrm{OH}} \mathrm{CF}_{3}$

2-Diazo-3,3,3-trifluoro-1-phenylpropan-1-ol (Table 1, 4g). Prepared from benzaldehyde $(3 \mathrm{~g}, 243 \mu \mathrm{L}, 2.4 \mathrm{mmol})$ according to the general procedure. Isolation by column chromatography afforded the title compound in $41 \%$ yield $(212.1 \mathrm{mg}, 0.98 \mathrm{mmol})$ as yellow oil. ${ }^{1} \mathrm{H}$ NMR $\left(300 \mathrm{MHz}, \mathrm{DMSO}-d_{6}\right) \delta 7.53-7.23(\mathrm{~m}$, $5 \mathrm{H}), 6.46(\mathrm{~d}, J=5.5 \mathrm{~Hz}, 1 \mathrm{H}), 5.63(\mathrm{~d}, J=5.5 \mathrm{~Hz}, 1 \mathrm{H}) .{ }^{13} \mathrm{C} \mathrm{NMR}(75 \mathrm{MHz}, \mathrm{DMSO}) \delta 140.72$, $128.49,126.71(\mathrm{q}, J=268.7 \mathrm{~Hz}), 126.18,66.88,57.28(\mathrm{~d}, J=41.0 \mathrm{~Hz}) .{ }^{19} \mathrm{~F}$ NMR $(282 \mathrm{MHz}$, DMSO) $\delta$-55.86. HRMS (EI): $m / z$ : calcd for $\mathrm{C}_{9} \mathrm{H}_{7} \mathrm{OF}_{3}\left[\mathrm{M}-\mathrm{N}_{2}\right]^{+}: 188.0449$, found: 188.0453 .<smiles>N#CC(C(O)c1ccc(Br)cc1)C(F)(F)F</smiles>
1-(4-Bromophenyl)-2-diazo-3,3,3-trifluoropropan-1-ol (Table 1, 4h). Prepared from 4-bromobenzaldehyde (3h, $444.1 \mathrm{mg}, 2.4 \mathrm{mmol})$ according to the general procedure. Isolation by column chromatography afforded the title compound in $50 \%$ yield $(352.9 \mathrm{mg}, 1.20 \mathrm{mmol}$, contains 3 $\%$ starting material) as yellow solid, mp $43-45{ }^{\circ} \mathrm{C} .{ }^{1} \mathrm{H}$ NMR $\left(300 \mathrm{MHz}, \mathrm{DMSO}-d_{6}\right) \delta 7.61$ $(\mathrm{d}, J=8.5 \mathrm{~Hz}, 2 \mathrm{H}), 7.40(\mathrm{~d}, J=8.5 \mathrm{~Hz}, 2 \mathrm{H}), 6.55(\mathrm{~d}, J=5.5 \mathrm{~Hz}, 1 \mathrm{H}), 5.66(\mathrm{~d}, J=5.5 \mathrm{~Hz}$, 1H). ${ }^{13} \mathrm{C}$ NMR (75 MHz, DMSO) $\delta 140.23,131.86,128.52, \delta 126.60$ (q, $J=268.8 \mathrm{~Hz}$ ), 121.49, 66.38, 57.08 (q, $J=40.9 \mathrm{~Hz}) .{ }^{19} \mathrm{~F}$ NMR (282 MHz, DMSO) $\delta$-55.69. HRMS (EI): $m / z$ : calcd for $\mathrm{C}_{9} \mathrm{H}_{6} \mathrm{~N}_{2} \mathrm{OF}_{3} \mathrm{Br}[\mathrm{M}]^{+}:$293.9616, found: 293.9613 .<smiles>N#CC(O)C(c1ccccc1C(F)(F)F)C(F)(F)F</smiles>

\section{2-Diazo-3,3,3-trifluoro-1-(2-(trifluoromethyl)phenyl)propan-1-ol (Table}

1, 4i). Prepared from 2-(trifluoromethyl)benzaldehyde (3i, $317 \mu \mathrm{L}, 2.4$ mmol) according to the general procedure. Isolation by column chromatography afforded the title compound in $66 \%$ yield $(447.8 \mathrm{mg}, 1.58 \mathrm{mmol})$ as yellow solid, mp $36-38{ }^{\circ} \mathrm{C} .{ }^{1} \mathrm{H}$ NMR $\left(300 \mathrm{MHz}, \mathrm{DMSO}-d_{6}\right) \delta 7.95-7.86(\mathrm{~m}, 1 \mathrm{H}), 7.84-7.73(\mathrm{~m}$, $2 \mathrm{H}), 7.64-7.53(\mathrm{~m}, 1 \mathrm{H}), 6.77(\mathrm{~d}, J=5.4 \mathrm{~Hz}, 1 \mathrm{H}), 5.86(\mathrm{~d}, J=5.4 \mathrm{~Hz}, 1 \mathrm{H}) .{ }^{13} \mathrm{C}$ NMR $(75$ MHz, DMSO) $\delta 138.66,133.54,129.48,128.60,126.55$ (q, $J=5.8 \mathrm{~Hz}), \delta 126.38$ (q, $J=$ $269.0 \mathrm{~Hz}), 125.42$ (q, $J=30.3 \mathrm{~Hz}), 124.56(\mathrm{q}, J=273.9 \mathrm{~Hz}), 63.33,57.45(\mathrm{~d}, J=41.4 \mathrm{~Hz})$. ${ }^{19}$ F NMR (282 MHz, DMSO) $\delta-56.44,-58.39$. HRMS (EI): $m / z$ : calcd for $\mathrm{C}_{10} \mathrm{H}_{6} \mathrm{~N}_{2} \mathrm{OF}_{6}[\mathrm{M}]^{+}$: 284.0384, found: 284.0388 .<smiles>N#Cc1ccc(C(O)C(=N)C(F)(F)F)cc1</smiles>

4-(2-Diazo-3,3,3-trifluoro-1-hydroxypropyl)benzonitrile (Table 1, 4j). Prepared from 4-formylbenzonitrile (3j, $314.7 \mu \mathrm{L}, 2.4 \mathrm{mmol})$ according to the general procedure. Isolation by column chromatography afforded the title compound in $66 \%$ yield $(379.8 \mathrm{mg}, 1.57 \mathrm{mmol}$, contains $2 \%$ starting material) as yellow solid, mp $75-77{ }^{\circ} \mathrm{C} .{ }^{1} \mathrm{H}$ NMR $\left(300 \mathrm{MHz}, \mathrm{DMSO}-d_{6}\right) \delta 7.89$ 
$(\mathrm{d}, J=8.2 \mathrm{~Hz}, 2 \mathrm{H}), 7.64(\mathrm{~d}, J=8.2 \mathrm{~Hz}, 2 \mathrm{H}), 6.71(\mathrm{~d}, J=5.3 \mathrm{~Hz}, 1 \mathrm{H}), 5.79(\mathrm{~d}, J=5.3 \mathrm{~Hz}$, $1 \mathrm{H}) .{ }^{13} \mathrm{C}$ NMR (75 MHz, DMSO) $\delta 146.32,132.98,127.33,126.52$ (q, $J=268.8 \mathrm{~Hz}$ ), 119.06, 111.20, 66.52, 57.06 (q, $J=41.7 \mathrm{~Hz}) .{ }^{19} \mathrm{~F}$ NMR (282 MHz, DMSO) $\delta-55.55$. HRMS (EI): $m / z$ : calcd for $\mathrm{C}_{10} \mathrm{H}_{6} \mathrm{~N}_{3} \mathrm{OF}_{3}[\mathrm{M}]^{+}:$241.0463, found: 241.0457 .

Long Run Experiments. For feed A 2,2,2-trifluoroethylamine hydrochloride (1, 6.78 g, 50 mmol) was dissolved in water $(100 \mathrm{~mL}, 0.5 \mathrm{M})$ and eight drops of concentrated $\mathrm{H}_{2} \mathrm{SO}_{4}$ were added. For feed B, $\mathrm{NaNO}_{2}(6.90 \mathrm{~g}, 100 \mathrm{mmol})$ was dissolved in water $(100 \mathrm{~mL}, 1 \mathrm{M})$. Feed A and $\mathrm{B}$ were pumped into a T-mixer at room temperature by two syringe pumps (Asia, Syrris). ${ }^{\mathrm{S} 1}$ at flow rates of $200 \mu \mathrm{L} \mathrm{min}{ }^{-1}$ each. The mixture went through a short PFA tubing then further through the inner tube of the commercially available tube-in-tube device (GAM II, Uniqsis $)^{\mathrm{S} 2}$ made out of gas permeable Teflon AF2400 (0.8 mm inner diameter, $2 \mathrm{~mL}$ ). The aqueous waste stream leaving the inner tube of the reactor was quenched into $2 \mathrm{M} \mathrm{H}_{2} \mathrm{SO}_{4}$ after passing a 7 bar back pressure regulator (Vapourtec). ${ }^{\mathrm{S} 3}$ The outer tube of the reactor made out of a gas impermeable perfluoroalkox polymer (1.6 mm inner diameter, $\sim 6 \mathrm{~mL})$ was connected to a third syringe pump carrying feed C (dry THF) at a flow rate of $100 \mu \mathrm{L} / \mathrm{min}$. The outlet of the outer tubing was connected to a first glass column (Omnifit, $6.6 \mathrm{~mm}$ i.d., $2.4 \mathrm{~mL}$ volume) containing the scavenger material followed by a second glass column (Omnifit, $6.6 \mathrm{~mm}$ i.d., $2.4 \mathrm{~mL}$ volume) which was packed with the respective polymer bound base. After around 30 min equilibration time, feed $\mathrm{C}$ was switched form pure solvent to a $0.2 \mathrm{M}$ solution of aldehyde (3a) in dry THF. The product stream was collected after depressurization in a 6 bar backpressure regulator. The conversion was determined by GC-FID analysis over a period of $6 \mathrm{~h}$.

\section{X-ray Crystallography}

All crystals suitable for single crystal X-ray diffractometry were removed from a vial and immediately covered with a layer of silicone oil. A single crystal was selected, mounted on a glass rod on a copper pin, and placed in the cold $\mathrm{N}_{2}$ stream provided by an Oxford Cryosystems cryostream. XRD data collection was performed for compound $\mathbf{4 j}$, on a Bruker APEX II diffractometer with use of Mo K $\alpha$ radiation $(\lambda=0.71073 \AA)$ and a CCD area detector. Empirical absorption corrections were applied using SADABS. ${ }^{\text {S4,S5 }}$ The structures were solved with use of either direct methods or the Patterson option in SHELXS and refined by the full-matrix least-squares procedures in SHELXL. ${ }^{\mathrm{S}, \mathrm{S} 7}$ Non-hydrogen atoms were 
refined anisotropically. Hydrogen atoms were located in calculated positions corresponding to standard bond lengths and angles. Hydrogen atom bonded to $\mathrm{O} 1$ was located in a difference map. CCDC 1443602 contain the supplementary crystallographic data for compound $\mathbf{4 j}$ respectively. These data can be obtained free of charge from The Cambridge Crystallographic Data Centre via www.ccdc.cam.ac.uk/data_request/cif.

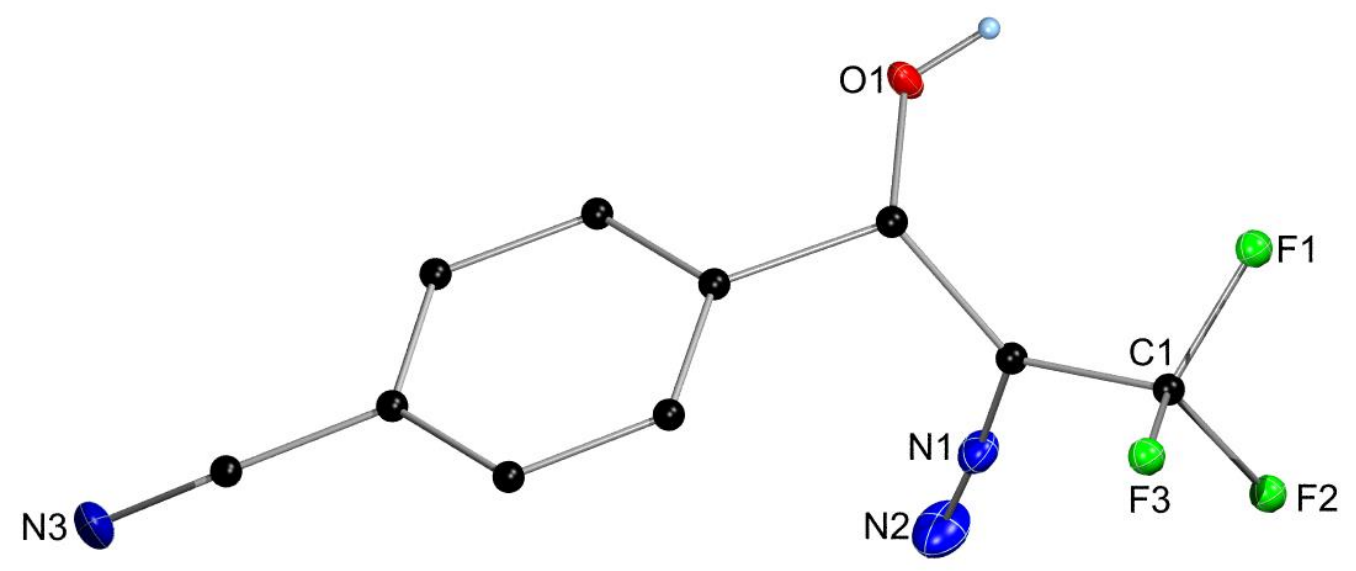

Figure S6. Crystal structure of $\mathbf{4 j}$. All non carbon atoms shown as $30 \%$ shaded ellipsoids.

\section{References}

S1 www.syrris.com

S2 http://www.uniqsis.com/

S3 www.vapourtec.com

S4 Bruker: APEX2 and SAINT. Bruker AXS Inc.: Madison, Wisconsin, USA, 2012.

S5 Blessing, R. Acta Crystallogr., Sect. A 1995, 51, 33-38.

S6 Sheldrick, G. Acta Crystallogr., Sect. A 1990, 46, 467-473.

S7 Sheldrick, G. A Acta Crystallogr., Sect. A 2008, 64, 112-122. 


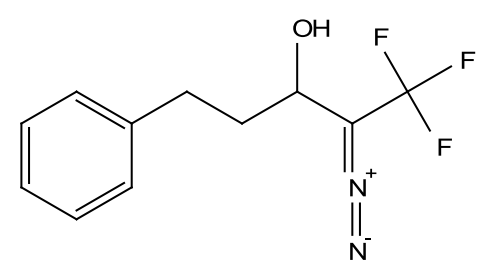

4a in DMSO-d ${ }_{6}$

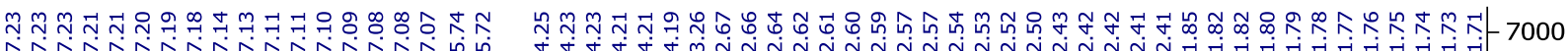
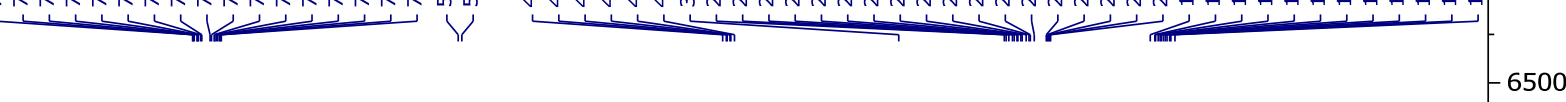

6000

$-5500$

5000

4500

$-4000$

3500

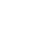

3000

2500

2000

$-1500$

1000

500 


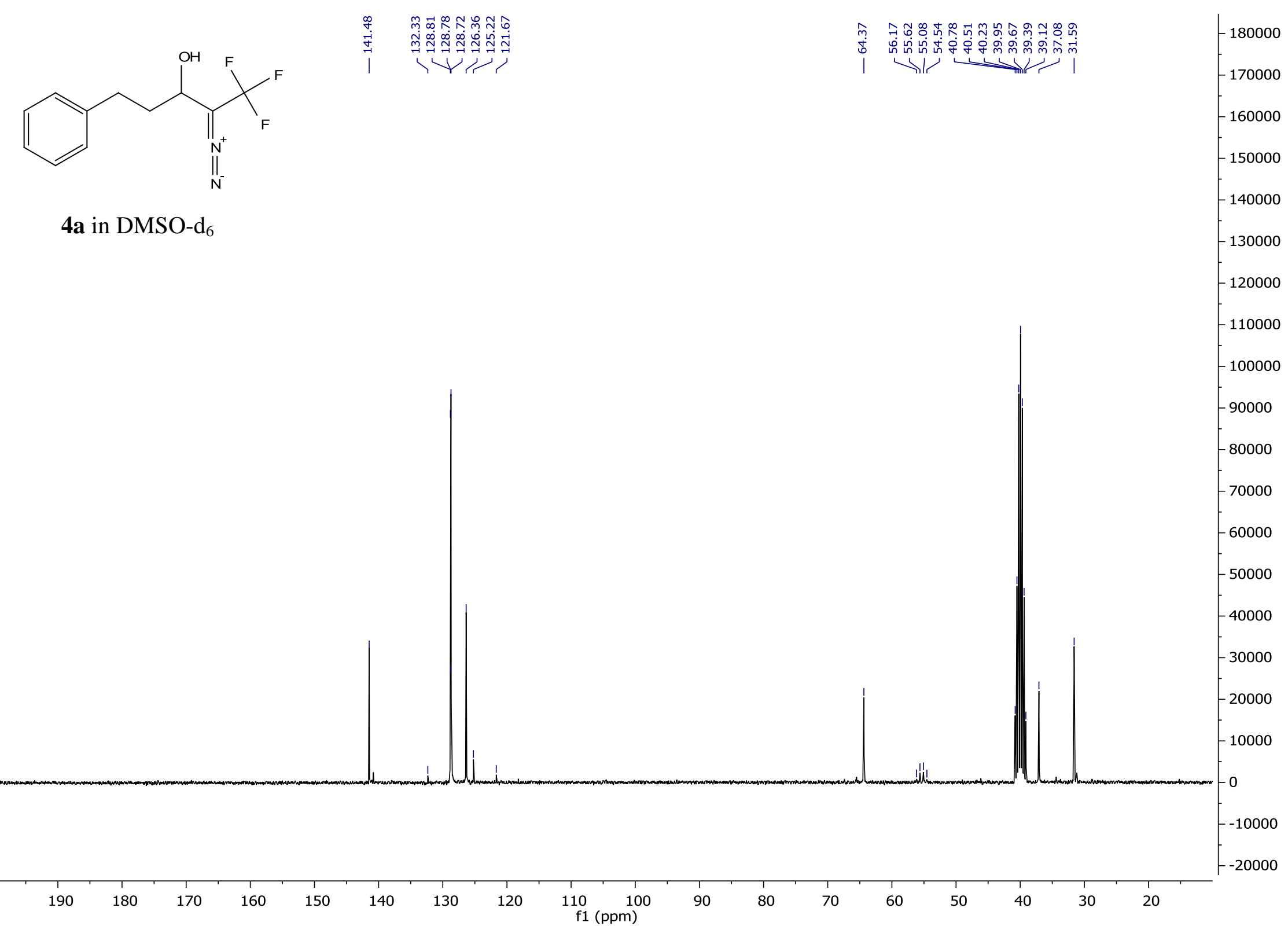




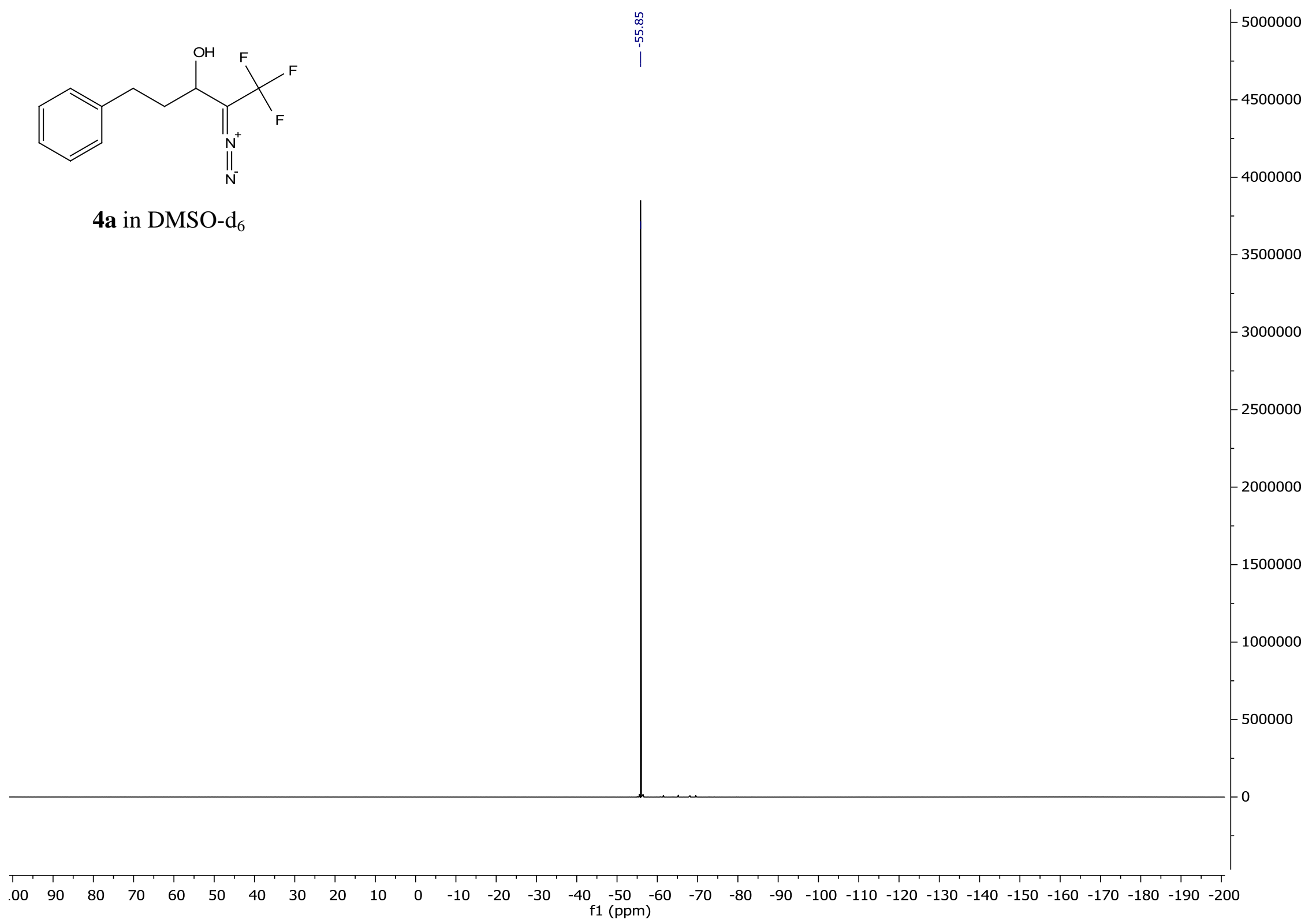




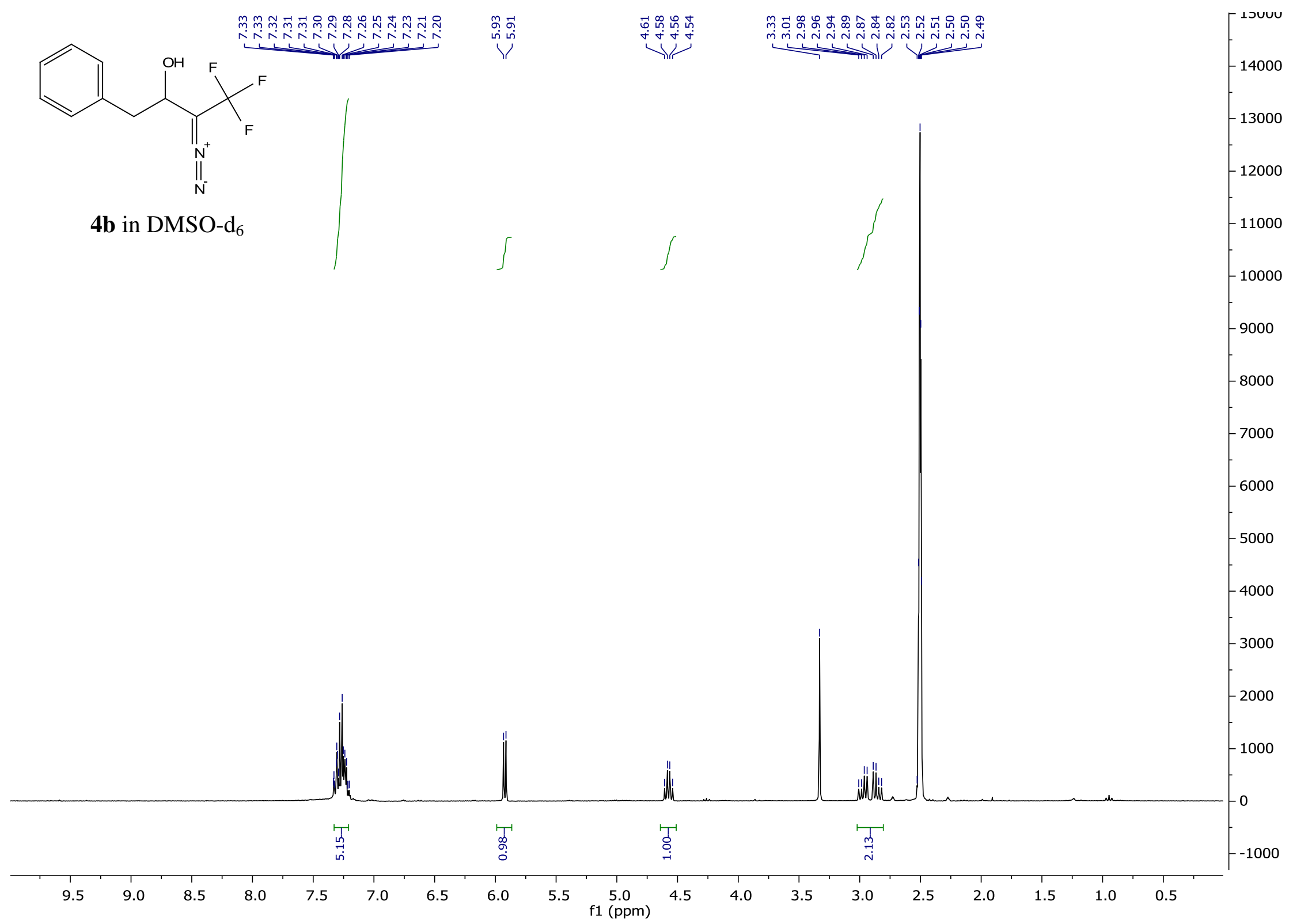




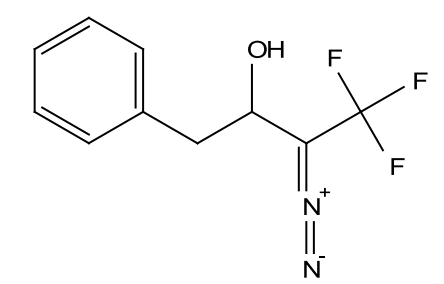

\%.

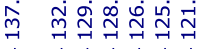

| |ा।

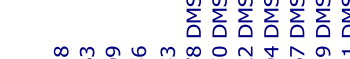

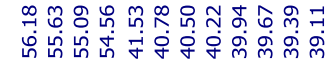

1II

4b in DMSO- $\mathrm{d}_{6}$

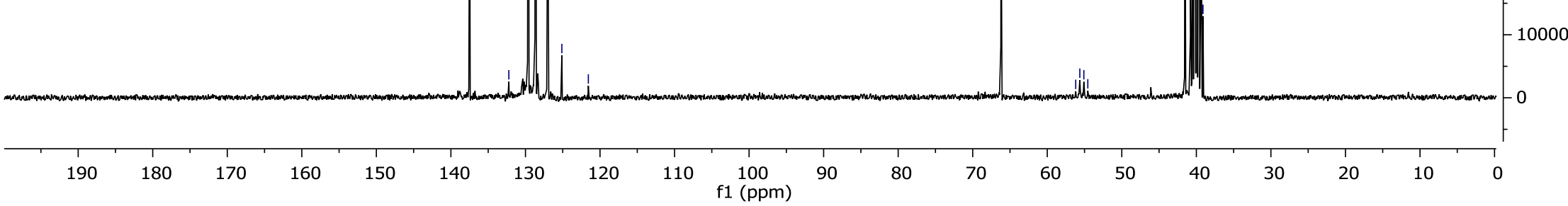




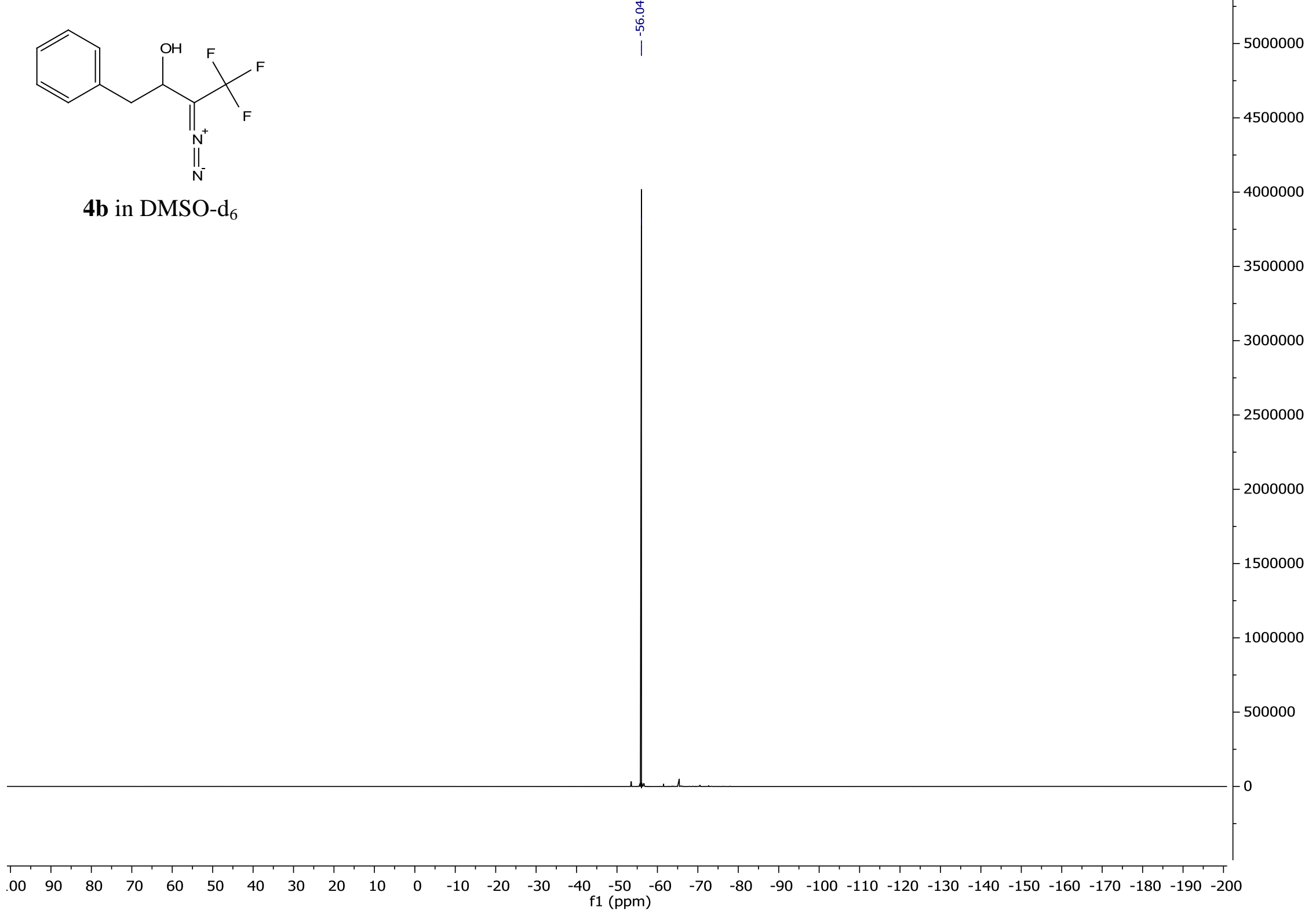




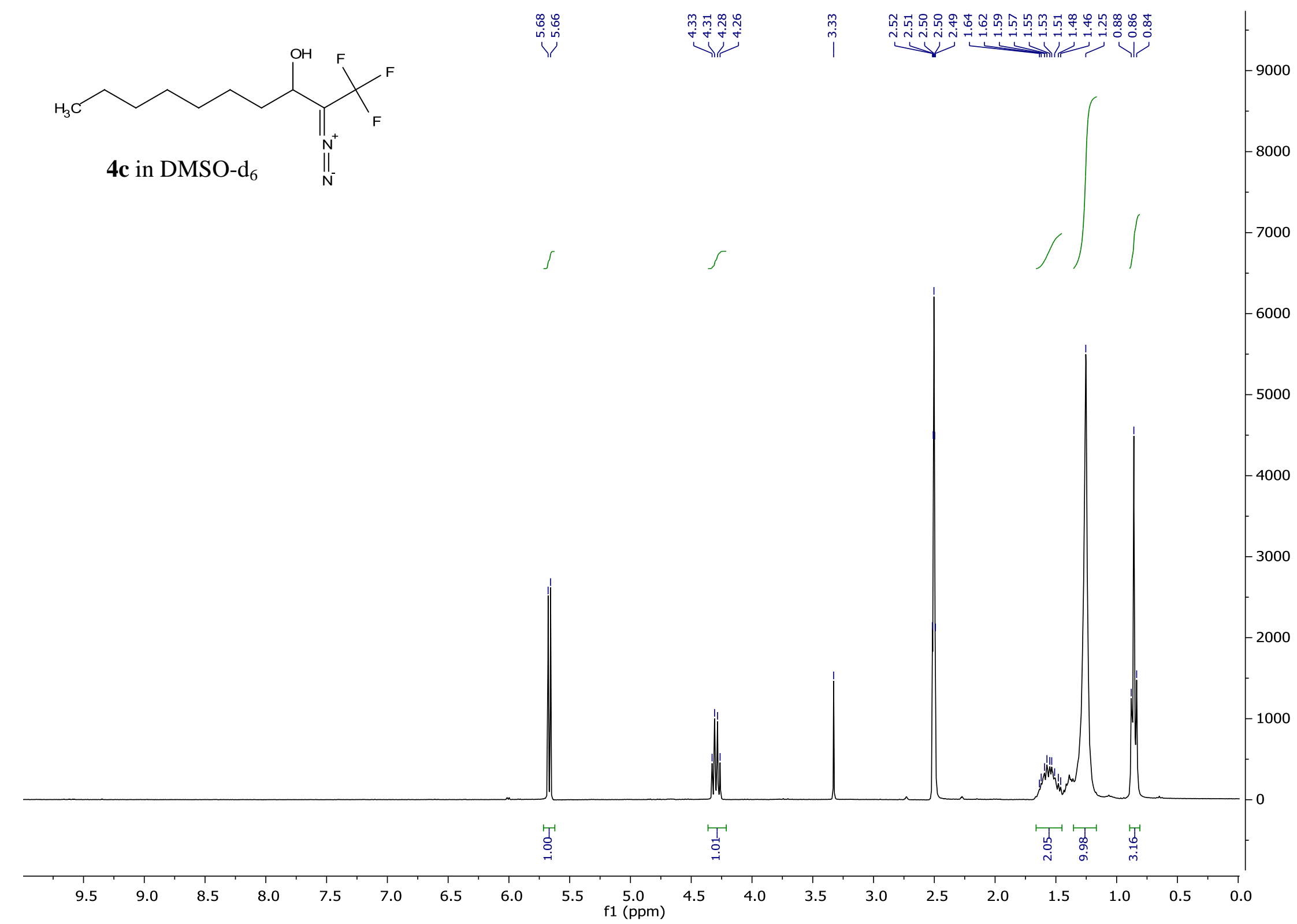




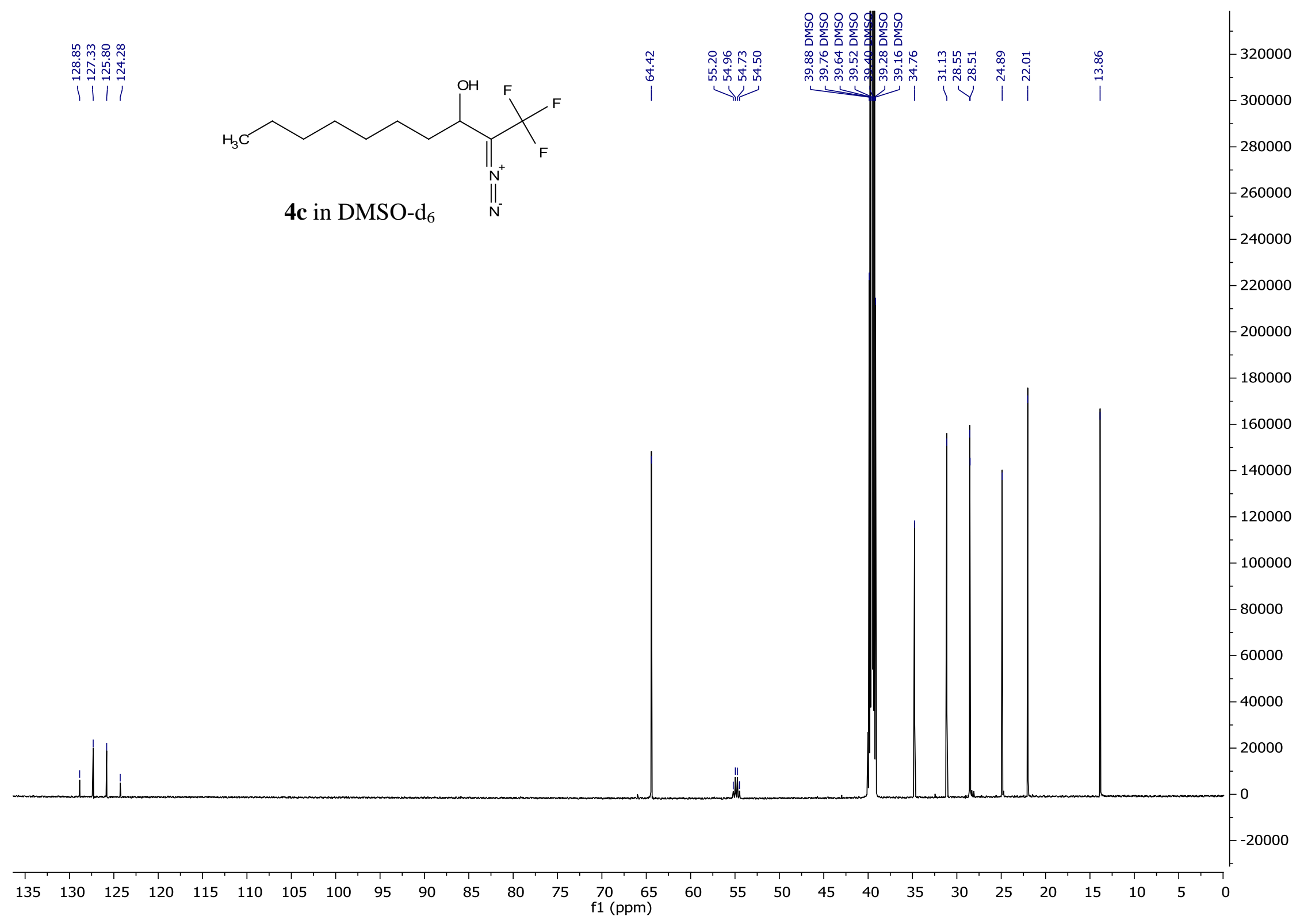




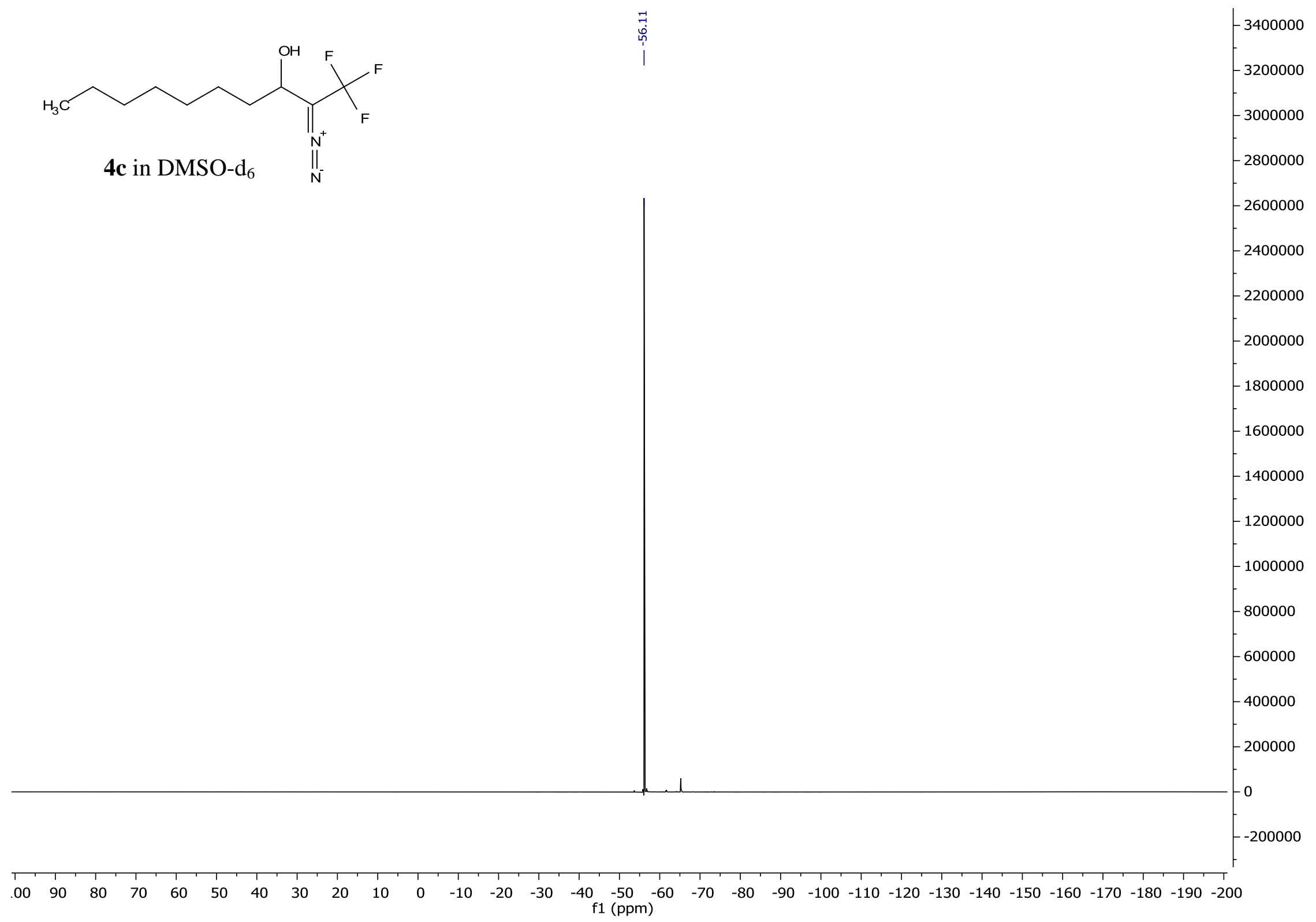




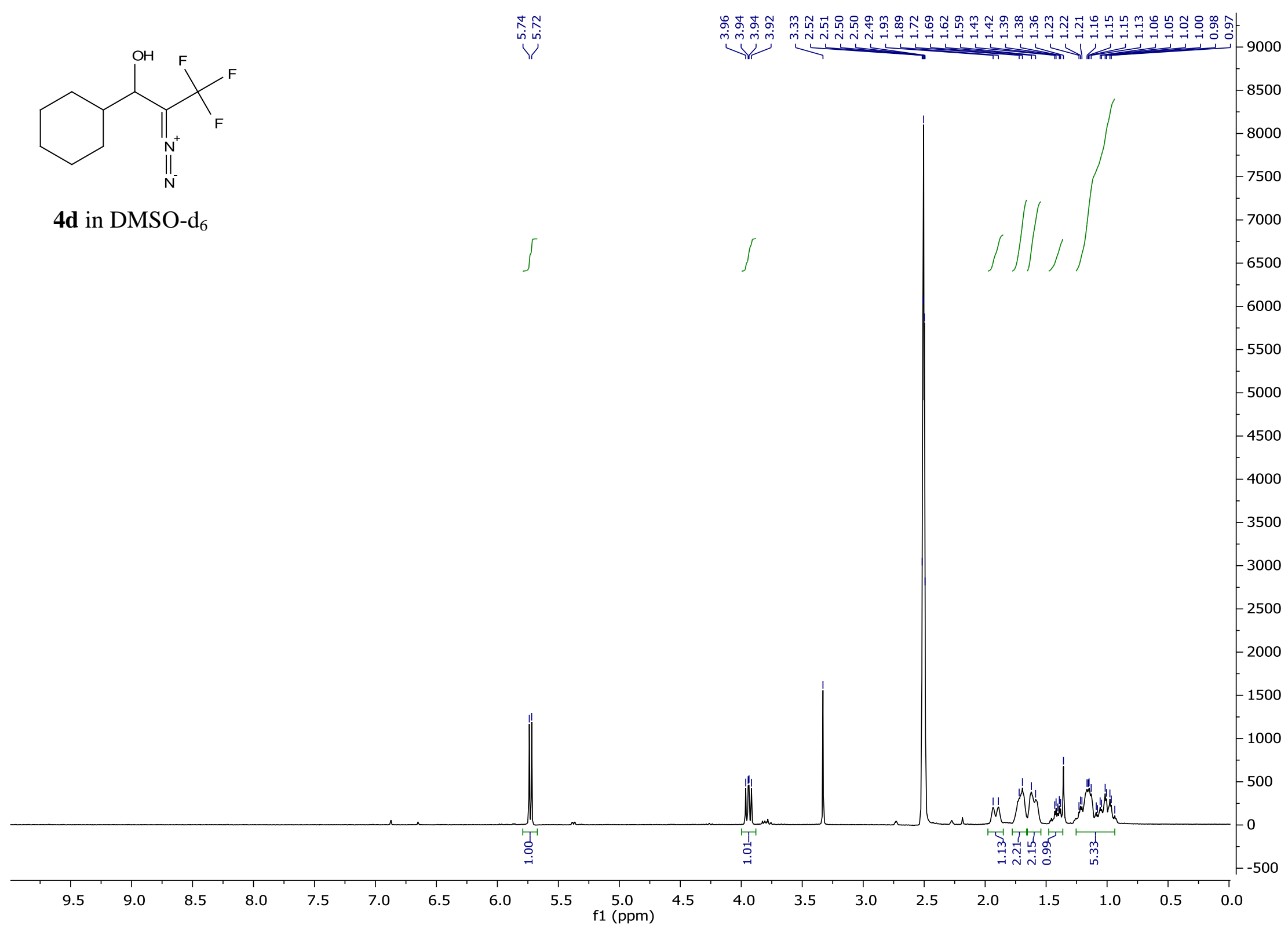




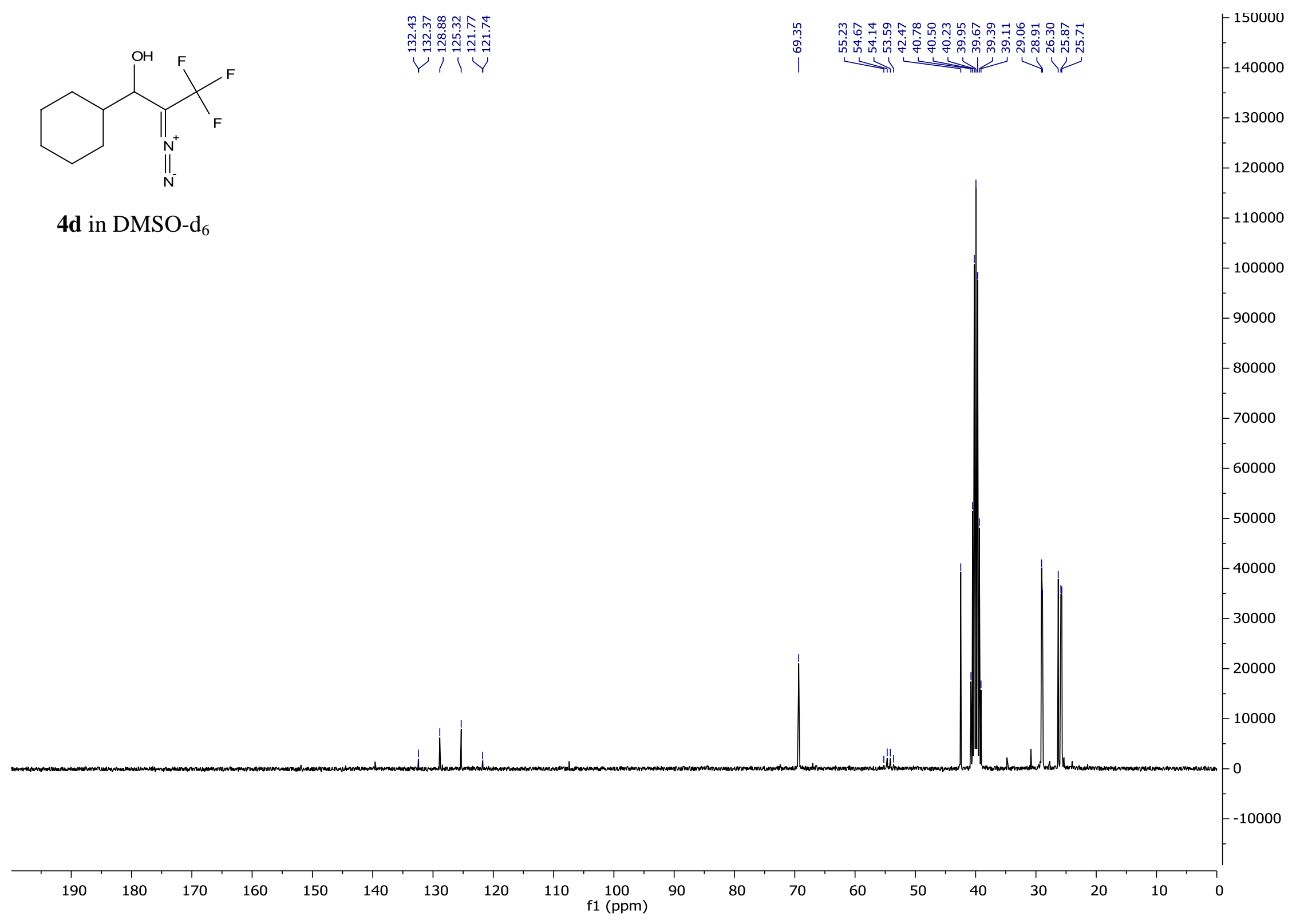




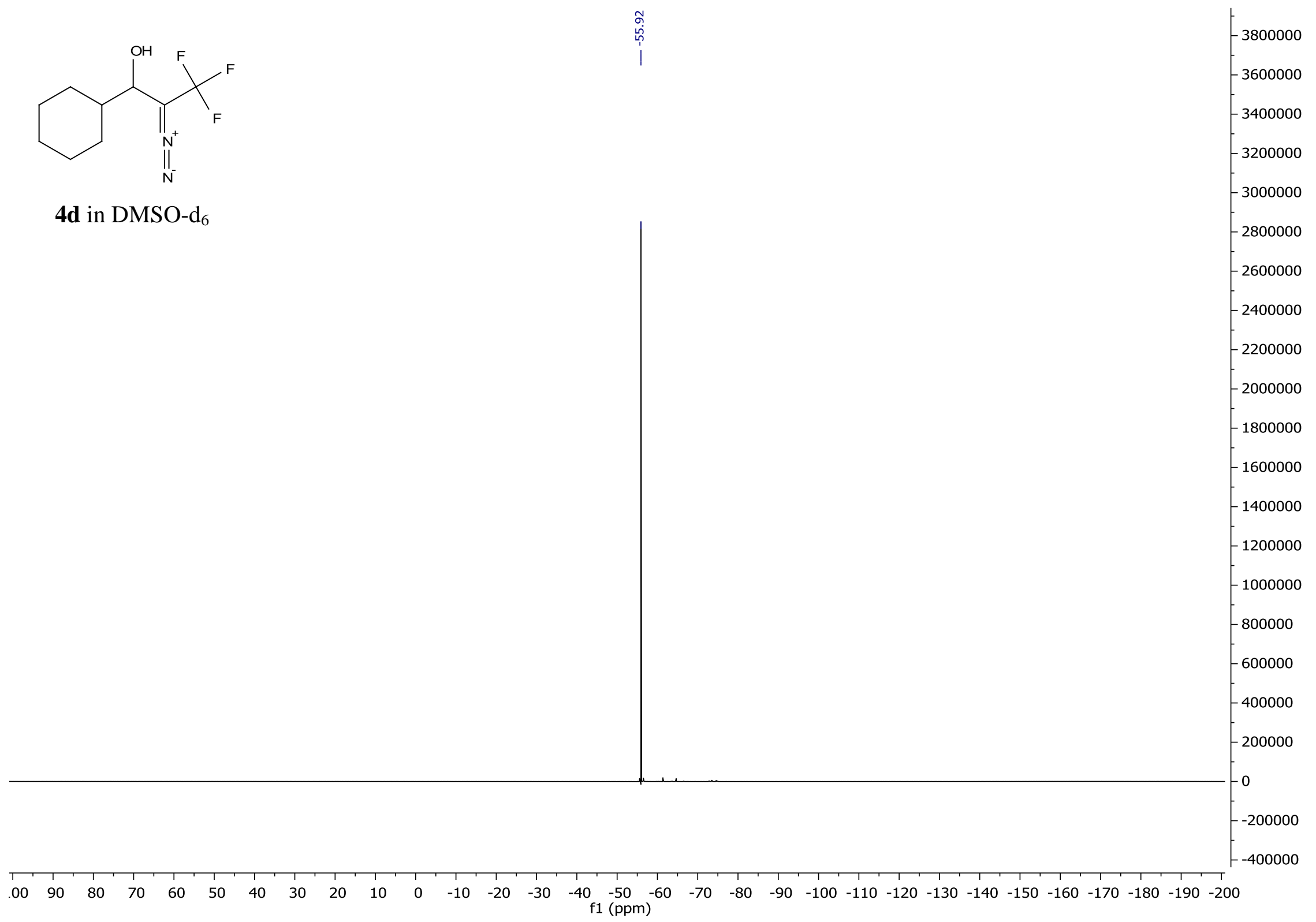




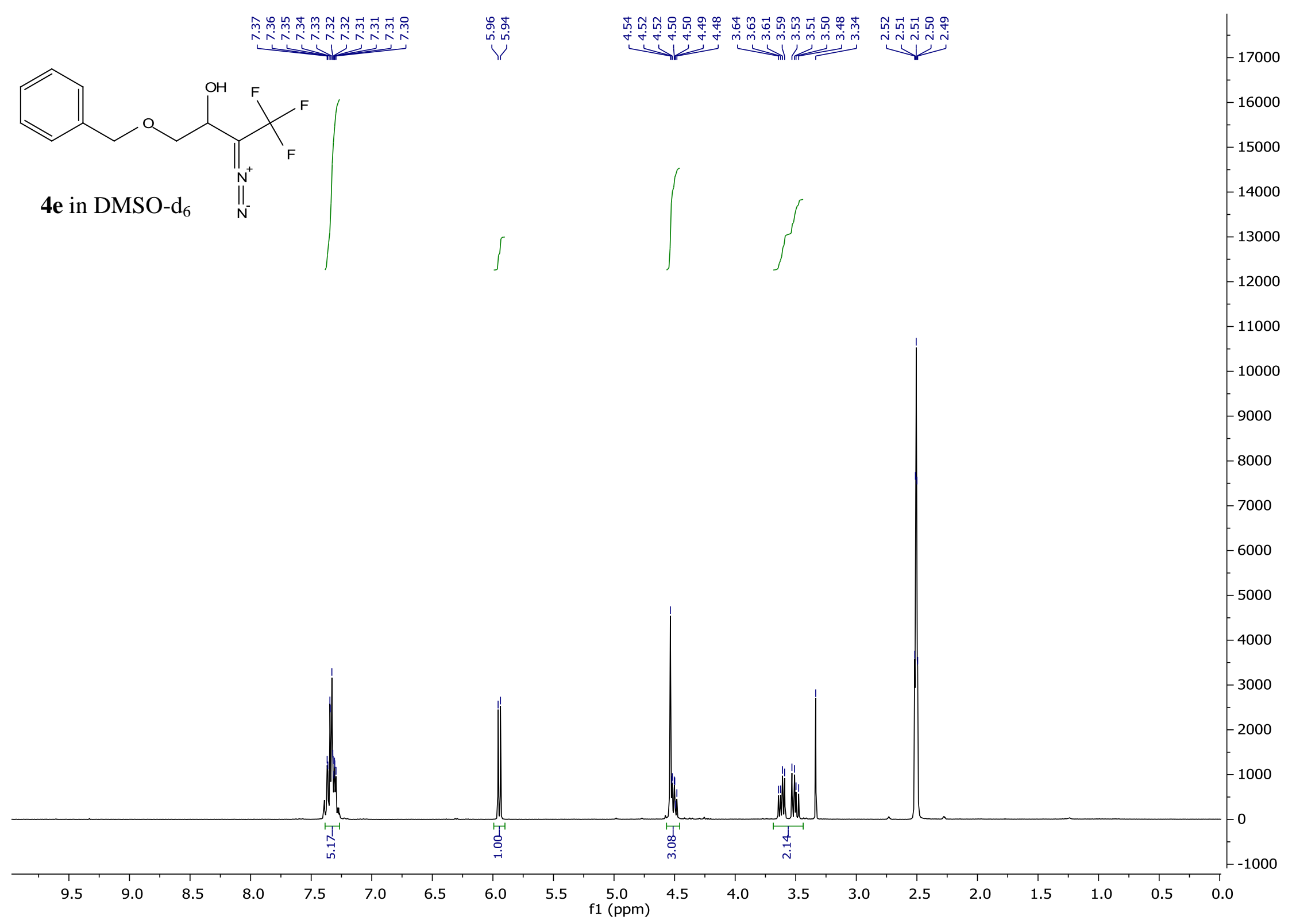




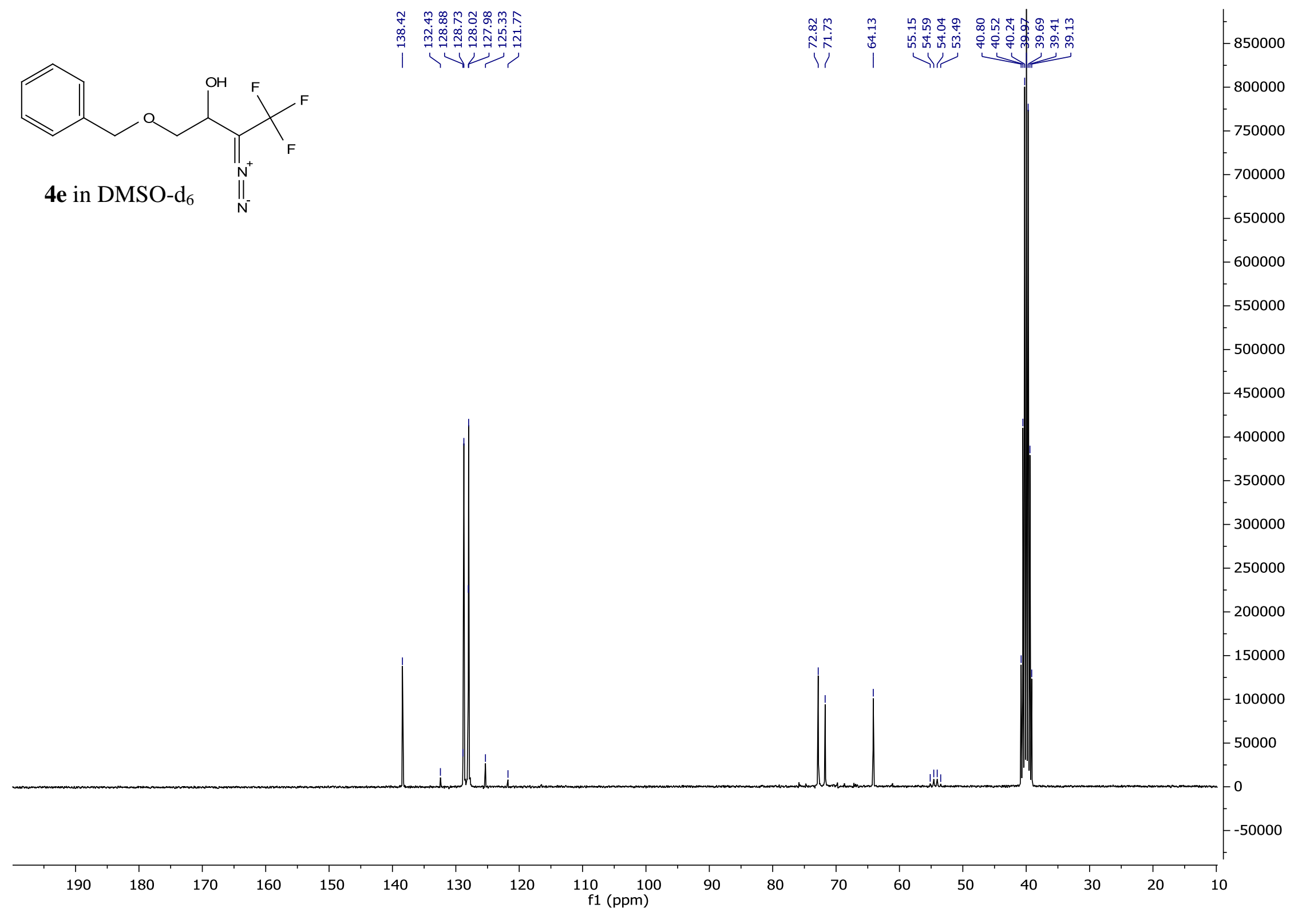




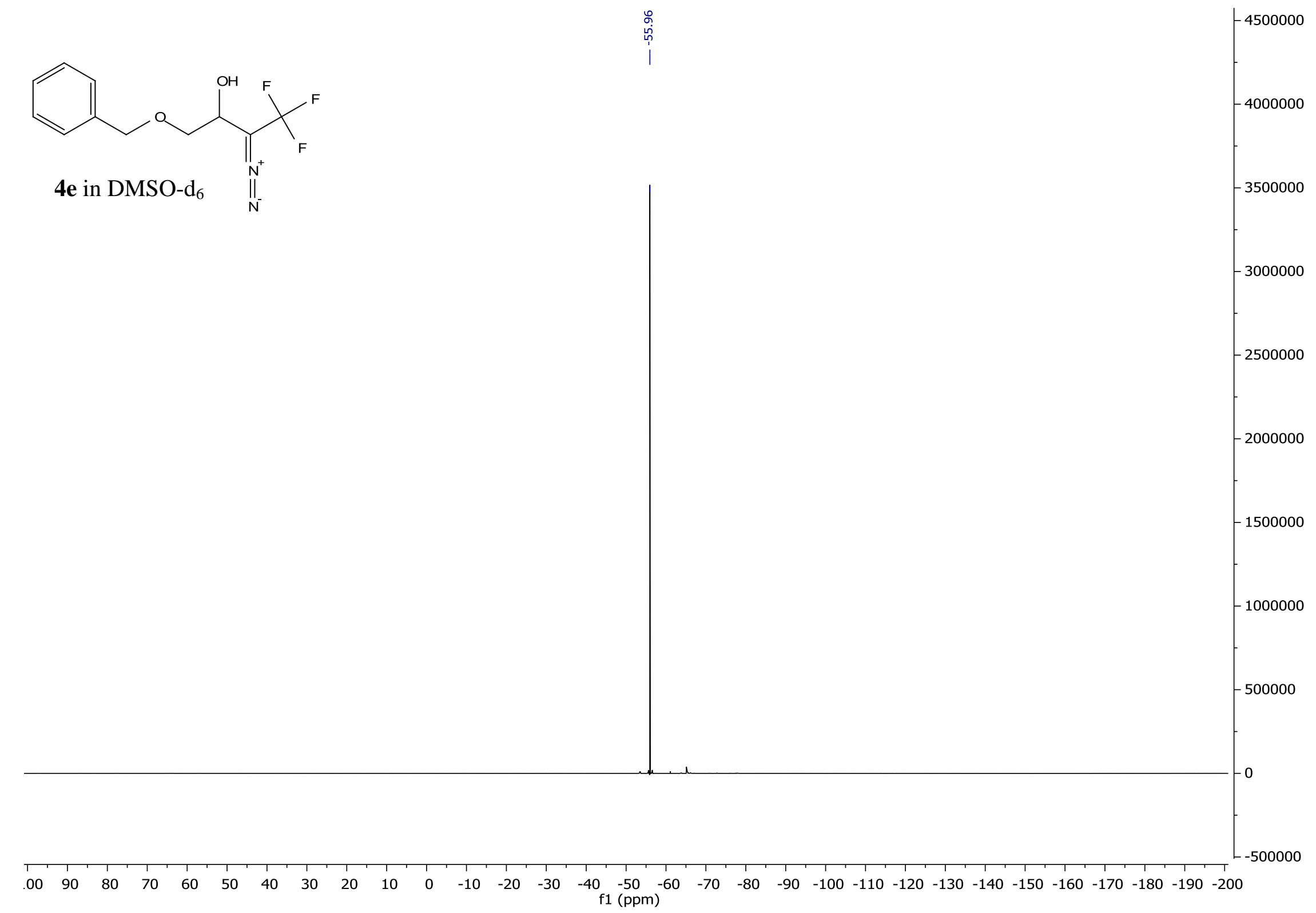




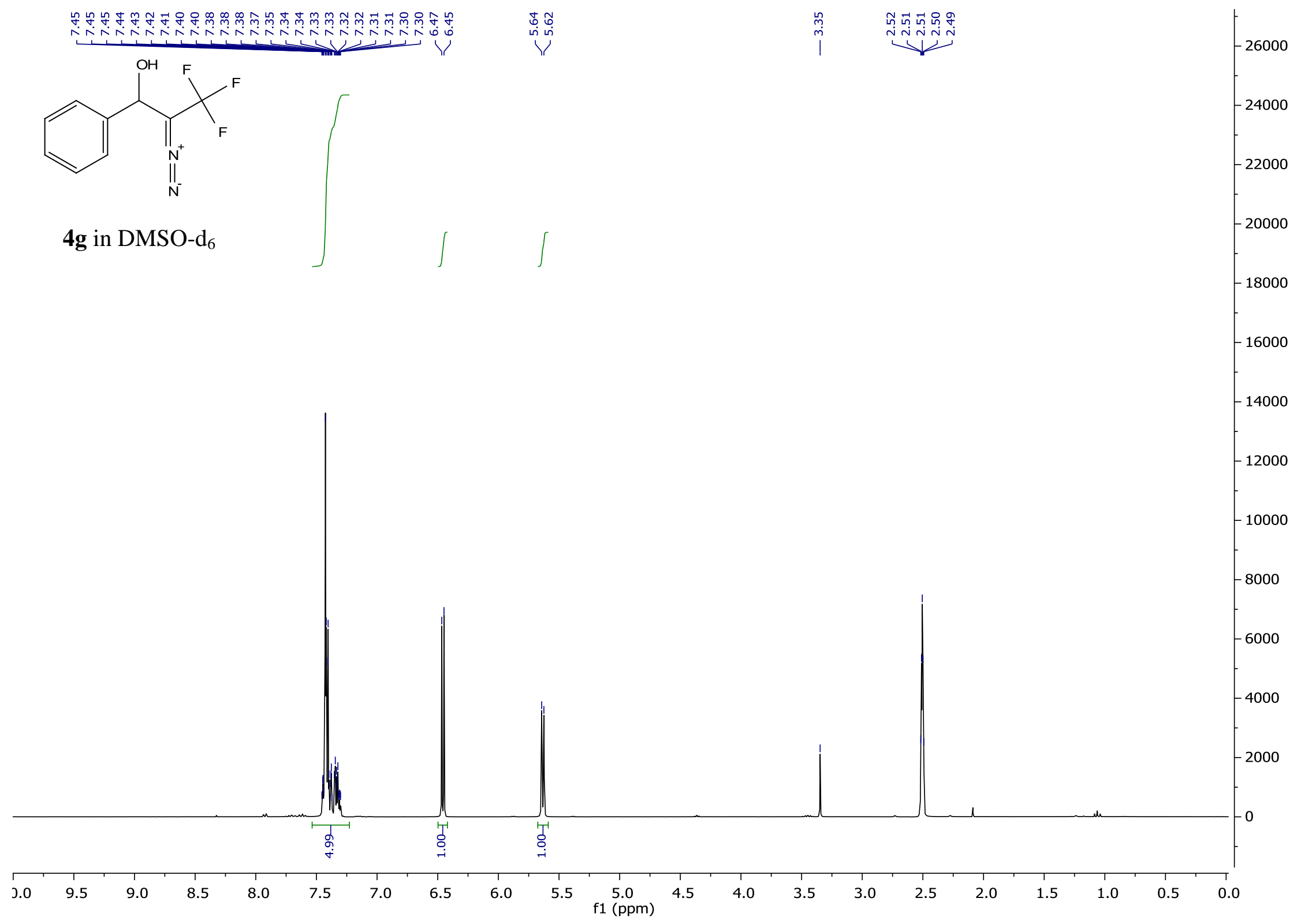




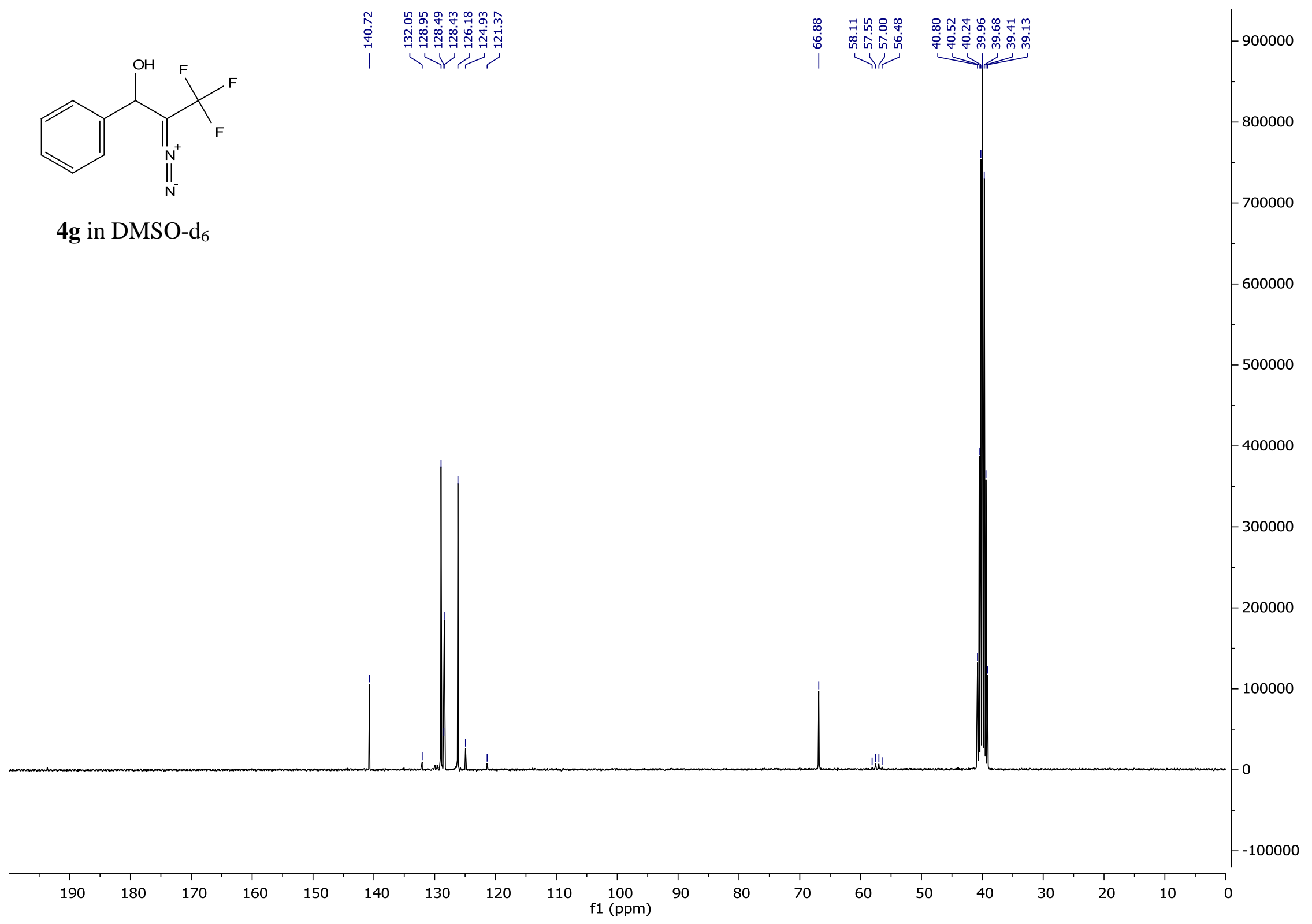




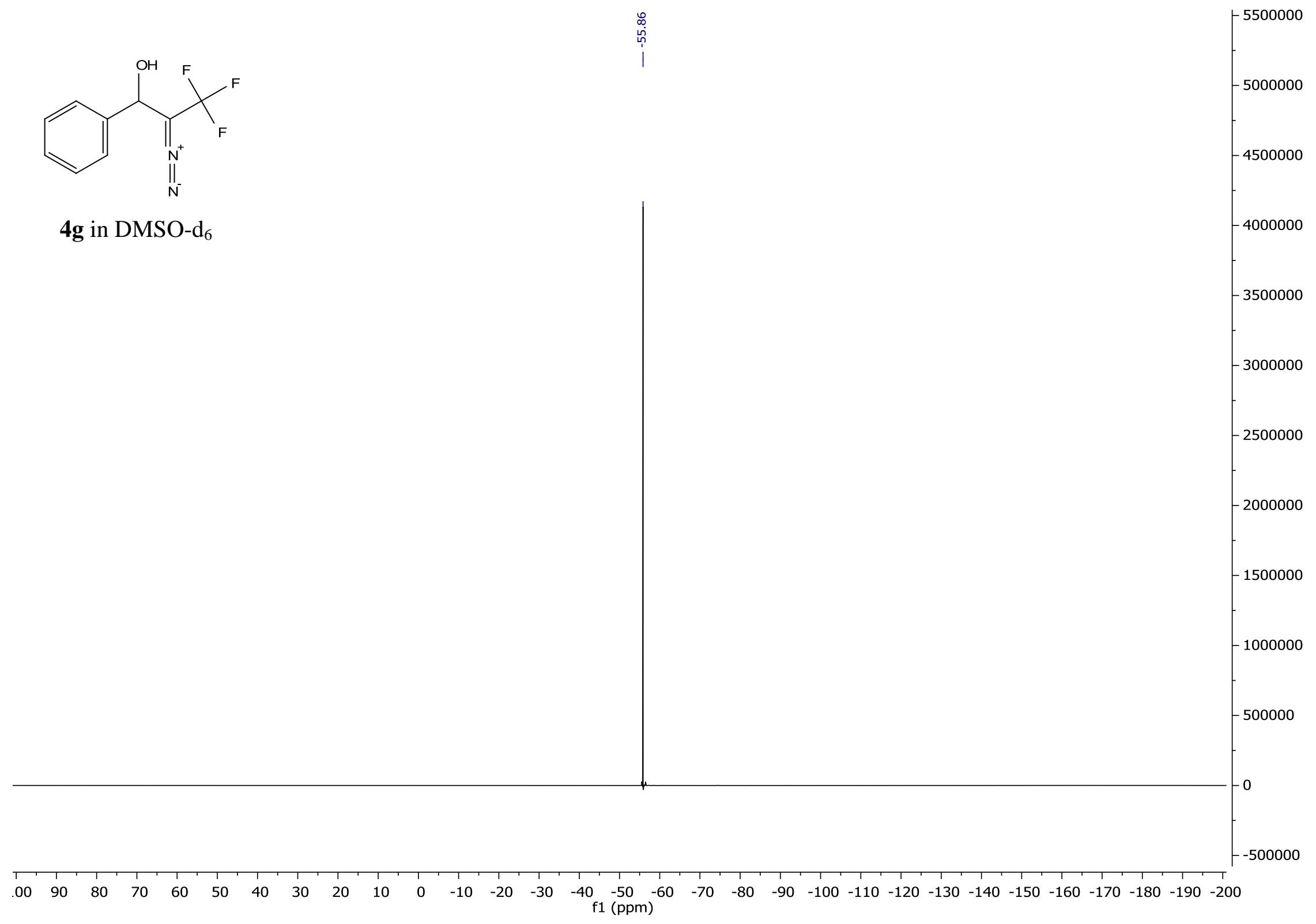




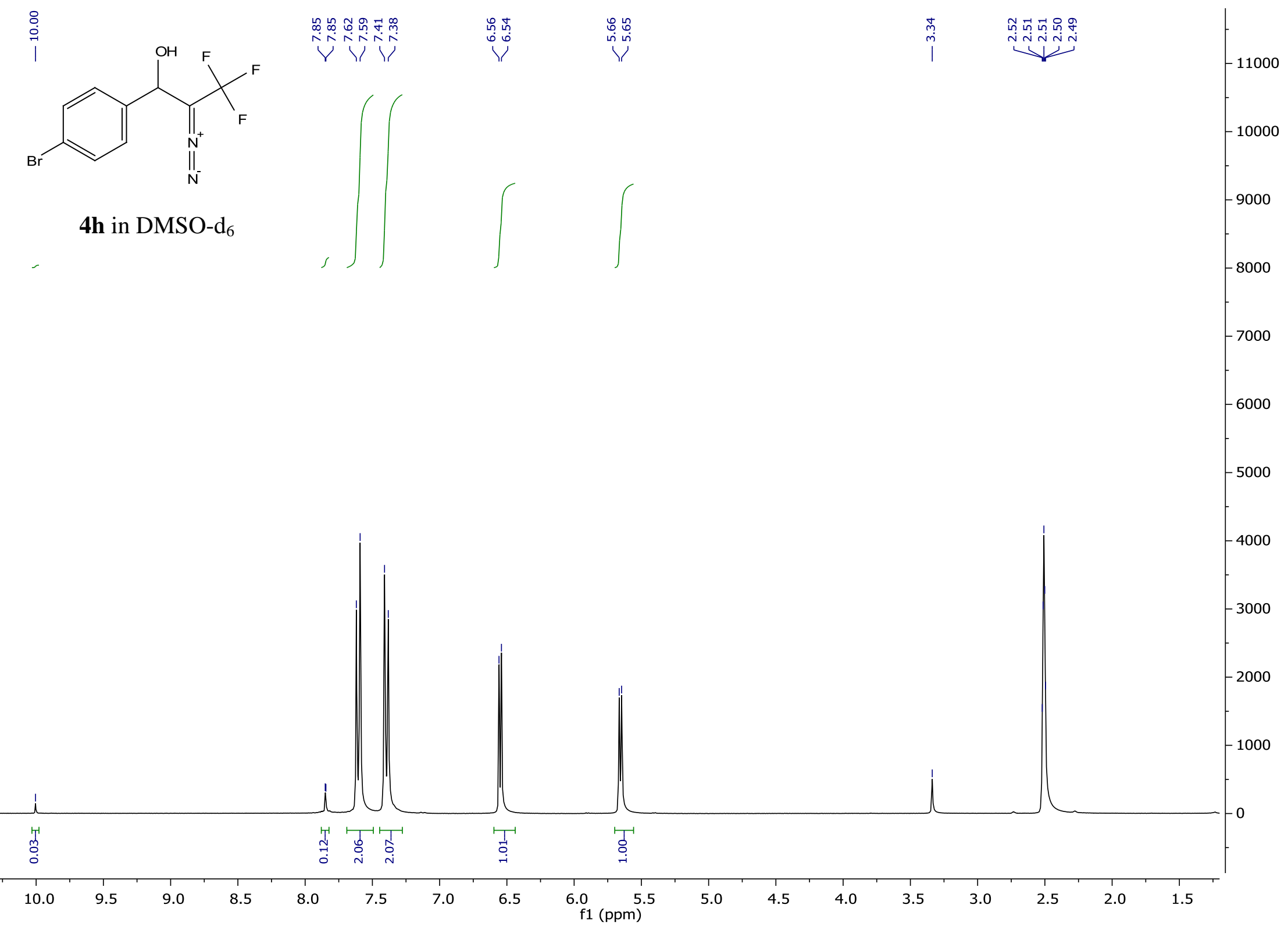




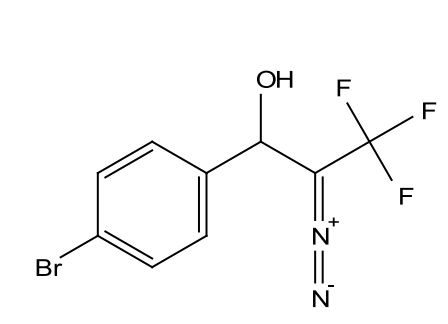

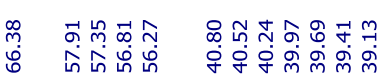

N

证

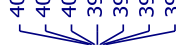

1400000

$-1300000$

1200000

$-1100000$

1000000

900000

800000

700000

600000

500000

400000

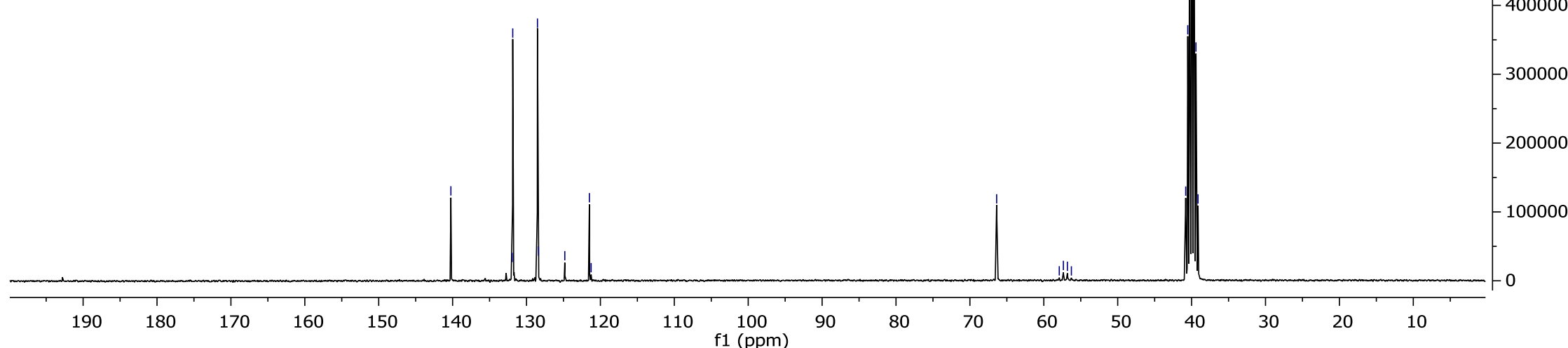



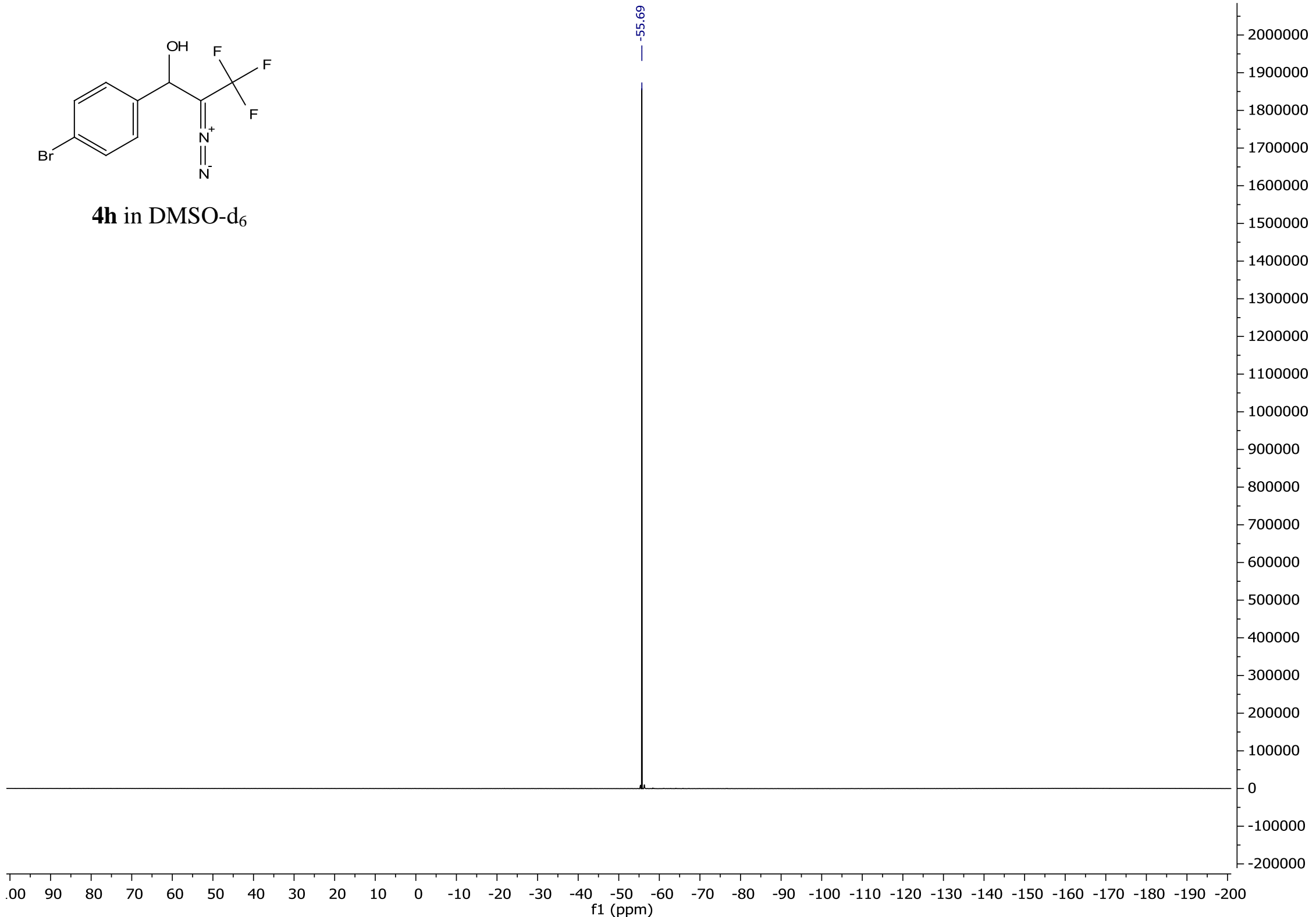

4h in DMSO-d 6 


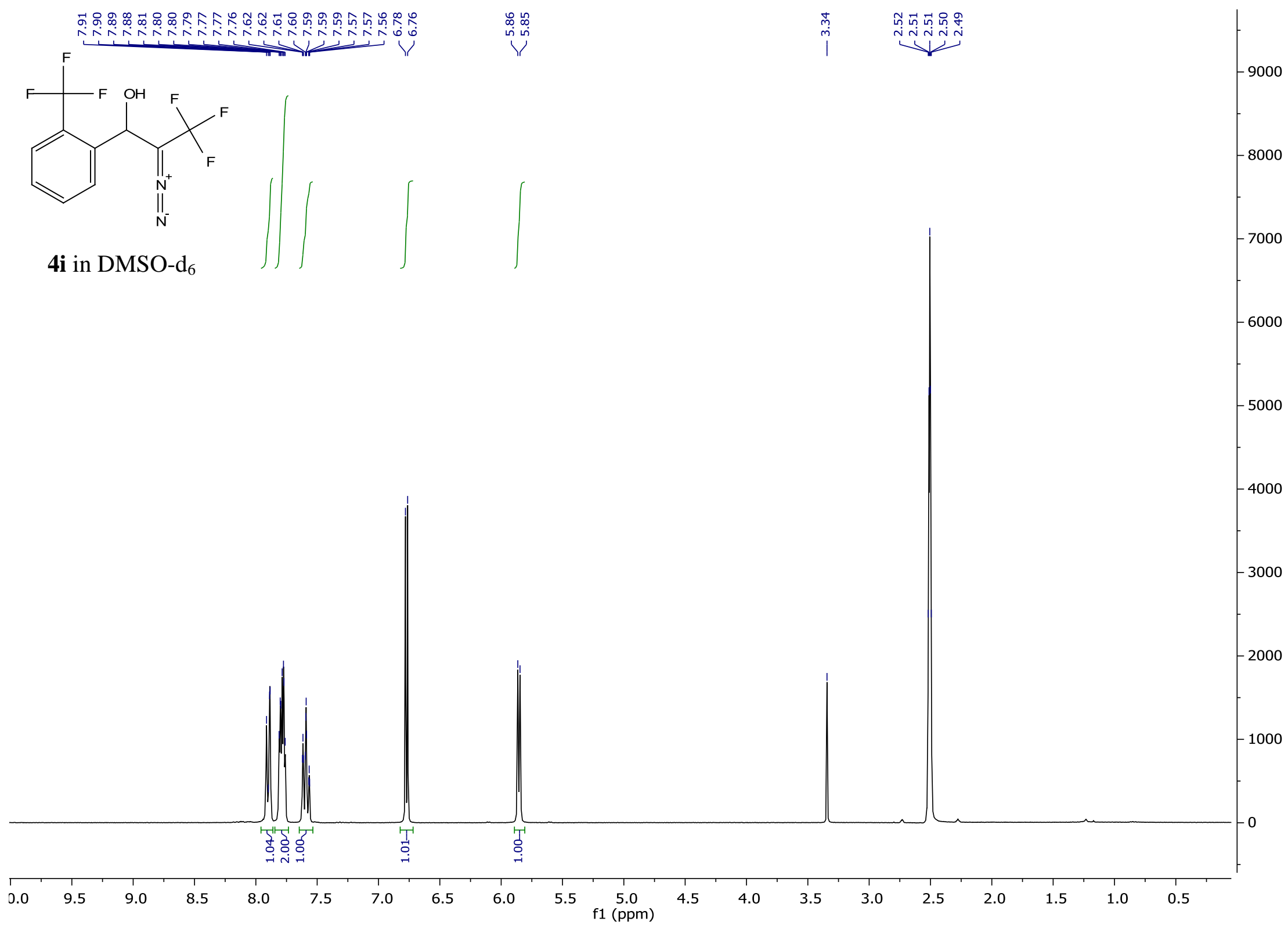




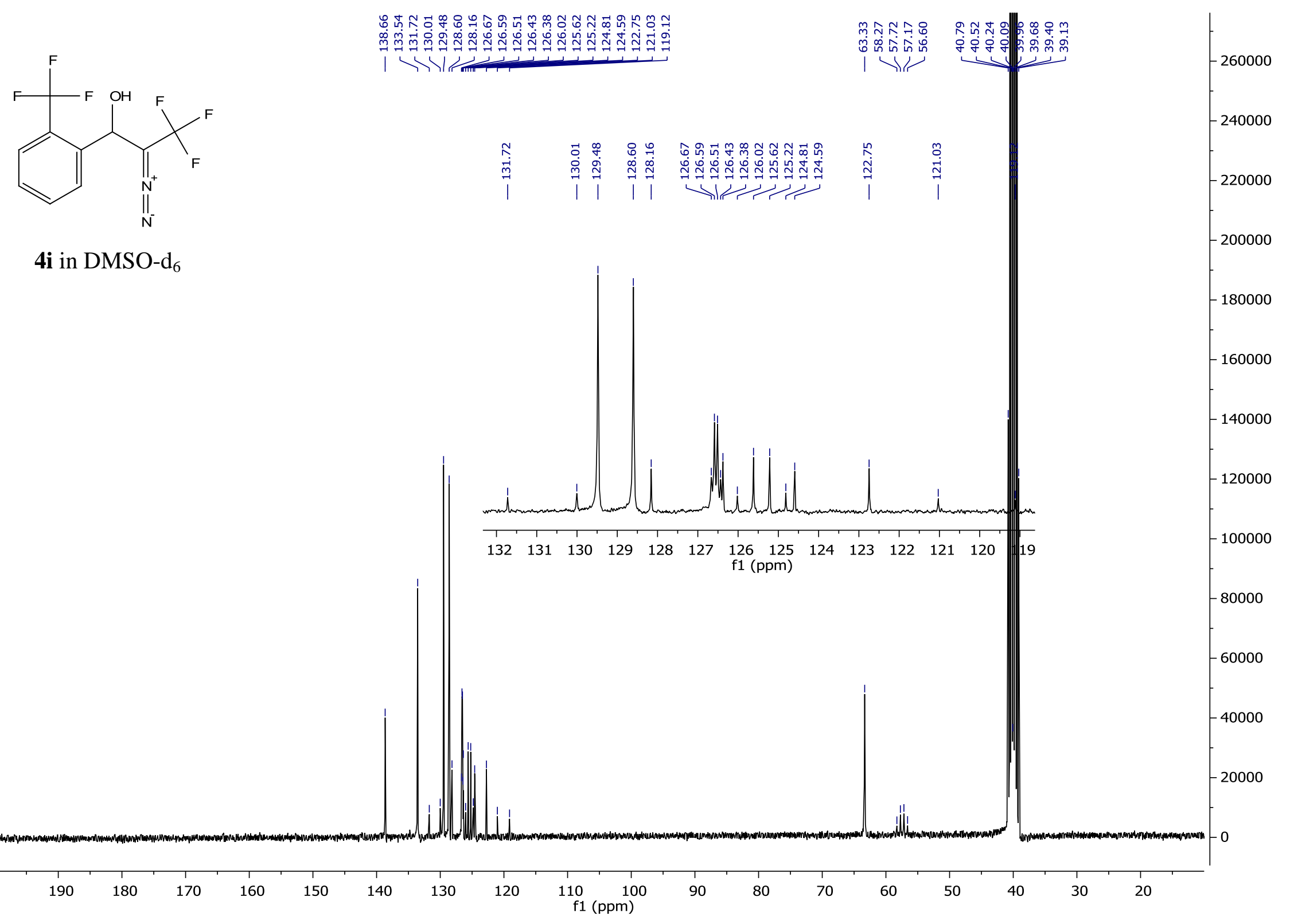




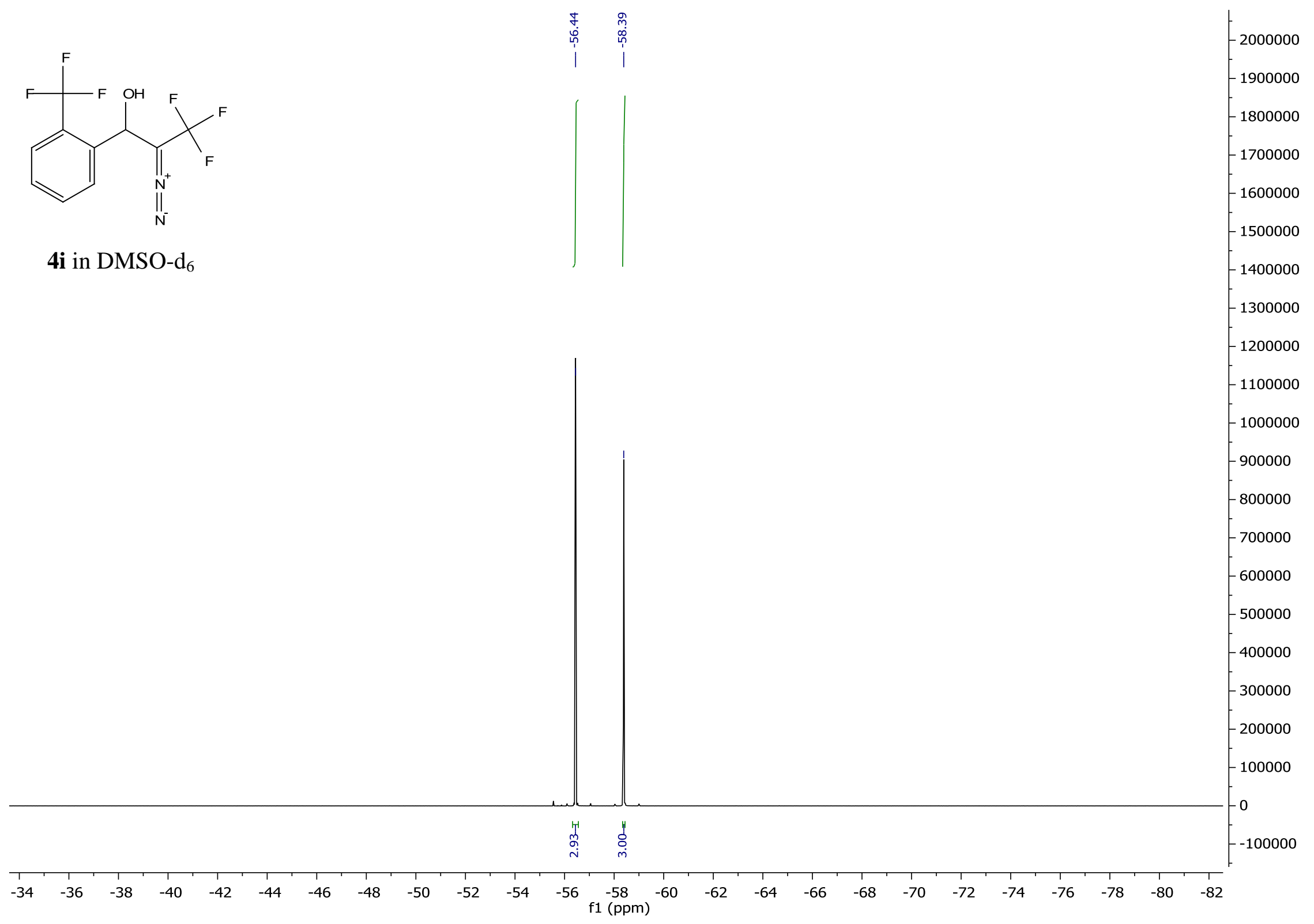




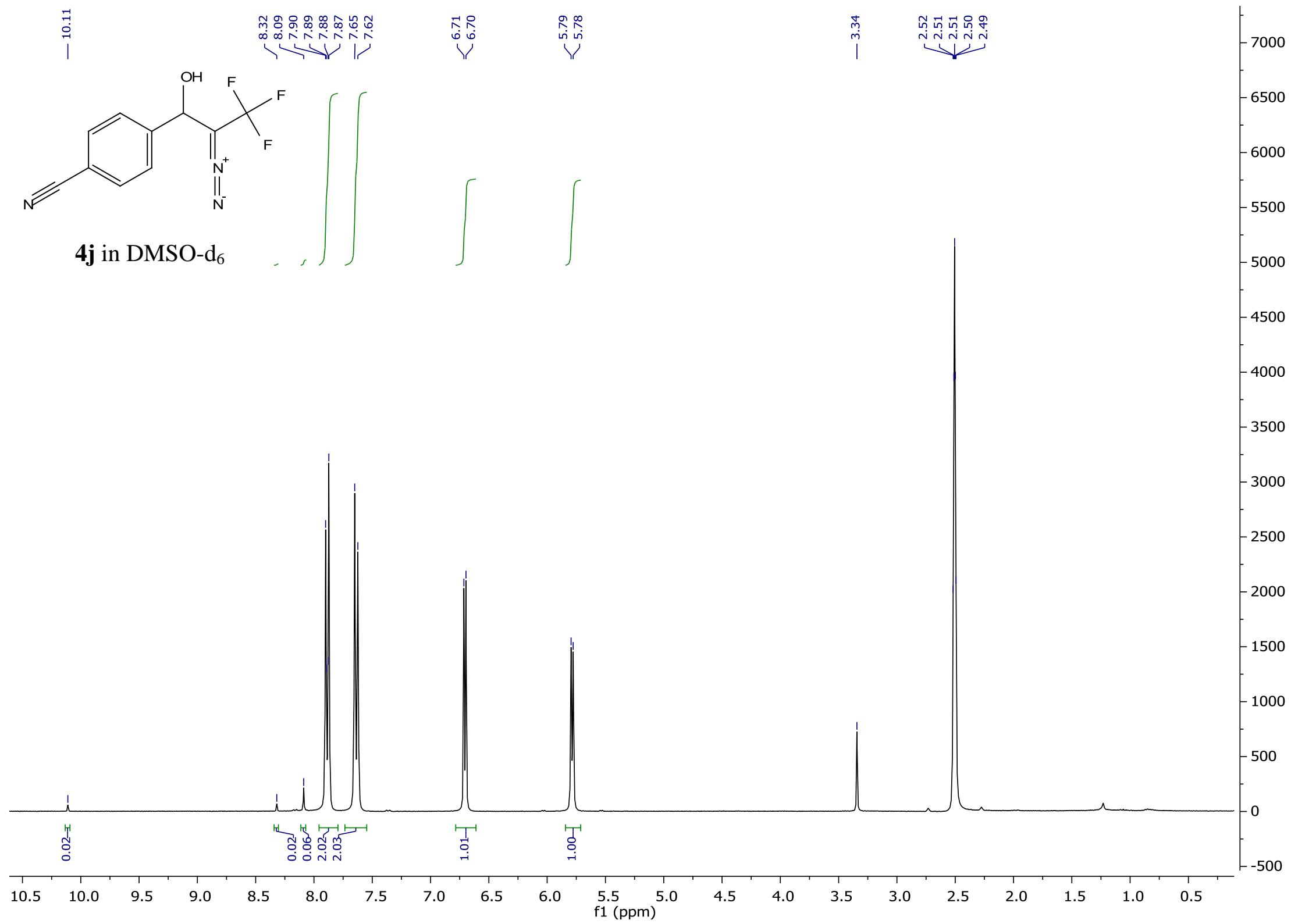




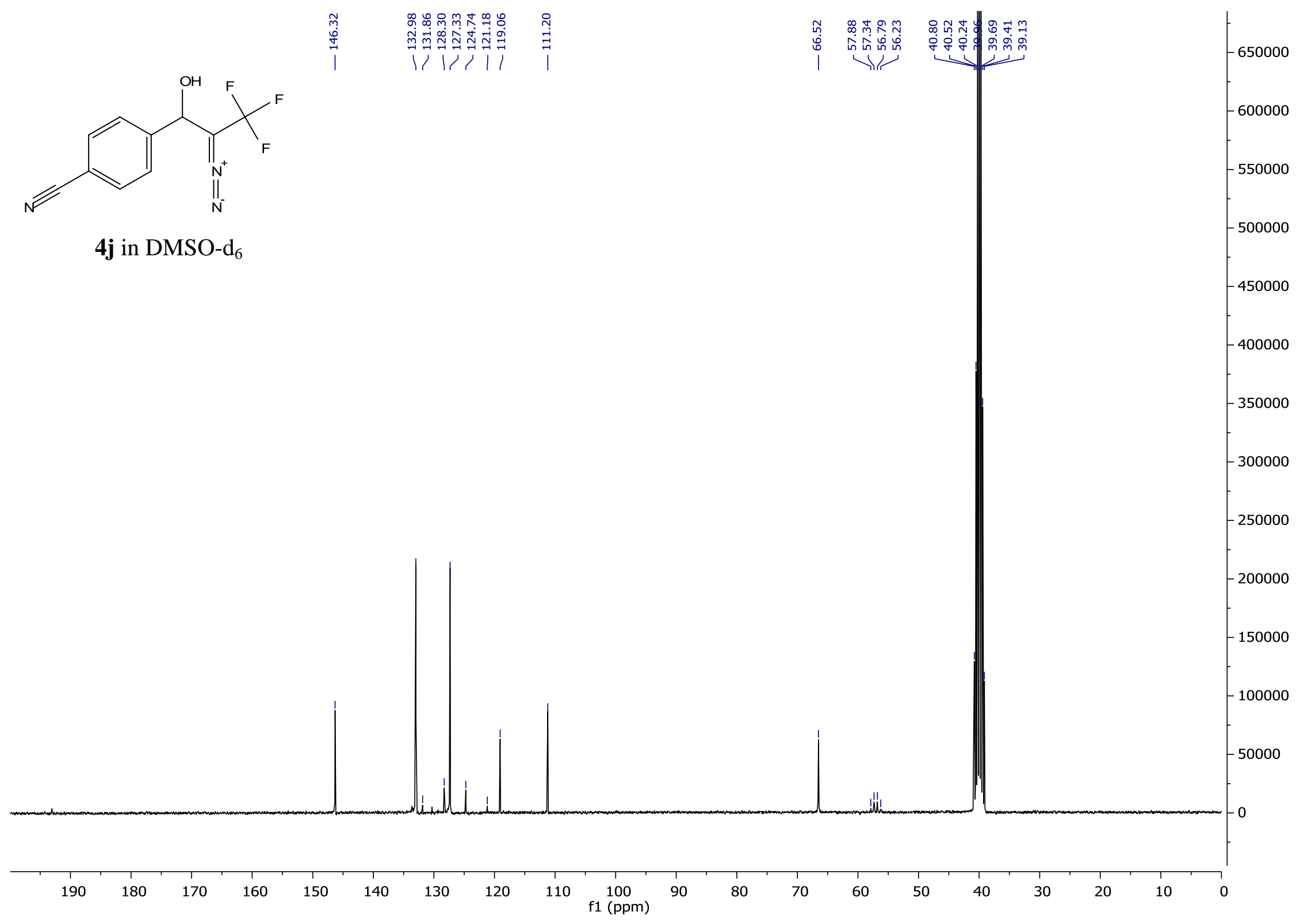




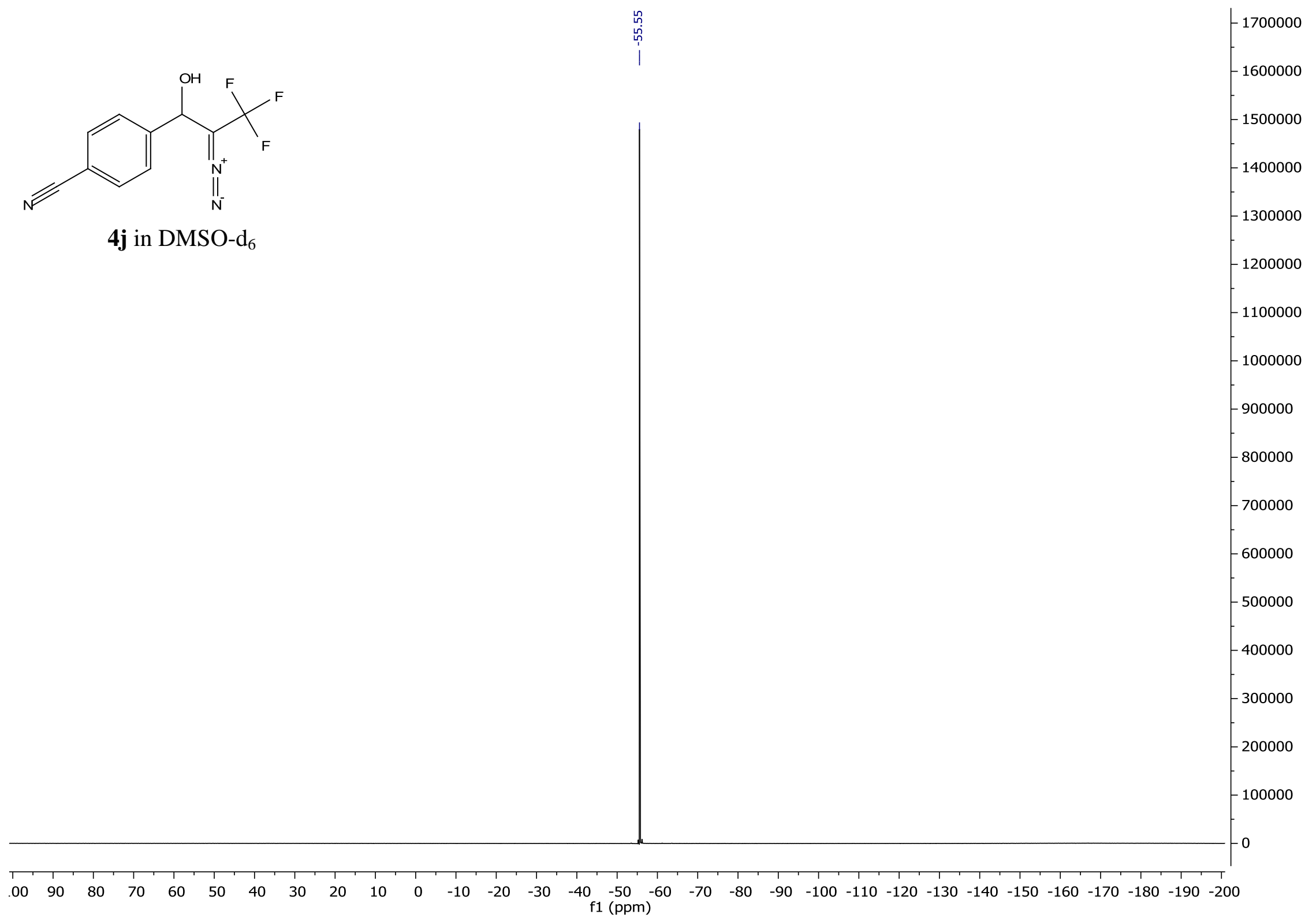

